\title{
From continuum mechanics to fracture mechanics: the strong discontinuity approach
}

\author{
J. Oliver * , A.E. Huespe, M.D.G. Pulido, E. Chaves \\ E.T.S. Enginyers de Camins, Canals i Ports. - Technical University of Catalonia (UPC), Campus Nord UPC (mòdul C-1) - Gran \\ Capitán s/n, 08034 Barcelona, Spain
}

\begin{abstract}
The paper deals with the strong discontinuity approach and shows the links with the decohesive fracture mechanics provided by that approach. On the basis of $1 \mathrm{D}$ continuum damage models it is shown that, by introducing some few ingredients like the strong discontinuity kinematics, discrete constitutive models (traction vs. displacement jumps) are automatically induced. For the general 2D-3D cases it is shown that the weak discontinuity concept is an additional ingredient, necessary in order to fulfill the strong discontinuity conditions, which allows to establish additional links with the fracture process zone concept. Also classical fracture mechanics properties as the fracture energy are related to the continuum model properties in a straightforward manner. (c) 2001 Elsevier Science Ltd. All rights reserved.
\end{abstract}

Keywords: Fracture; Localization; Strong discontinuities; Damage

\section{Introduction}

It is a very known fact that the observed mechanical behaviour of solids exhibits, beyond a limited elastic range, macroscopical discontinuities which, depending on the analysis environment, take the name of cracks, slip lines, shear bands etc. From the mechanical modelling point of view, those discontinuities can be characterized as jumps in the displacement field across material (fixed) lines and have been recently termed strong discontinuities [30]. Since very often the ultimate carrying capacity and the structural integrity itself are crucially affected by the appearance of such discontinuities, modelling the onset and propagation of strong discontinuities has become an important subject in solid mechanics. In this context the presently available approaches to model displacement discontinuities can be classified into two large groups, namely:

(1) The fracture mechanics approach: Linear fracture mechanics has been the classical tool to model displacement discontinuities. In addition, the necessity of dealing with the process of formation and propagation of discontinuities led the development of the non-linear (decohesive) fracture mechanics

\footnotetext{
${ }^{*}$ Corresponding author. Address: ETS Enginyers de Camins, Canals i Ports, Technical University of Catalonia (UPC), Campus Nord UPC (módul C-1), c/Gran Capitán s/n, 08034 Barcelona, Spain. Tel.: +34-93-4016490.

E-mail address: oliver@cimne.upc.es (J. Oliver).
} 
techniques essentially based on the introduction of adhoc discrete constitutive equations (tractions vs. displacement jumps) at one discontinuity interface inside an elastic continuum medium $[4,10]$.

(2) The continuum mechanics approach: It is well known that the inclusion, in standard continuum (stress vs. strain) non-linear constitutive equations, of strain-softening features (constitutive softening) leads to the strain-localization phenomenon, that is, the concentration of strains in narrow bands. This fact has frequently been envisaged as an appealing way to model displacement discontinuities (when the localization bands become narrower and narrower) [6]. However, it is also well known that in this case the boundary value problem become ill-conditioned resulting in instabilities and spurious bifurcations of the obtained solutions. Partial remedies have been found either by introducing mesh dependent regularizations of the continuum constitutive equation (smeared crack approach) [16,27] or performing substantial modifications on the nature of those equations (non-local models, viscous or gradient regularized models etc.) [15].

Both families of approaches are developed from different departure points and, frequently, in different research environments. This results in a lack of theoretical and fundamental connections between them. However, some attempts to link to each other have already been done [25] and the aim of this paper is to contribute to this field. The work outlines the general features of the so called strong discontinuity approach (SDA), which has been developed in the last years [1,13,14,17,18,20-22,24,26,29,30,32,33], whose main features are:

- The use of standard local continuum constitutive equations (stress-strain), non-linear and equipped with strain softening.

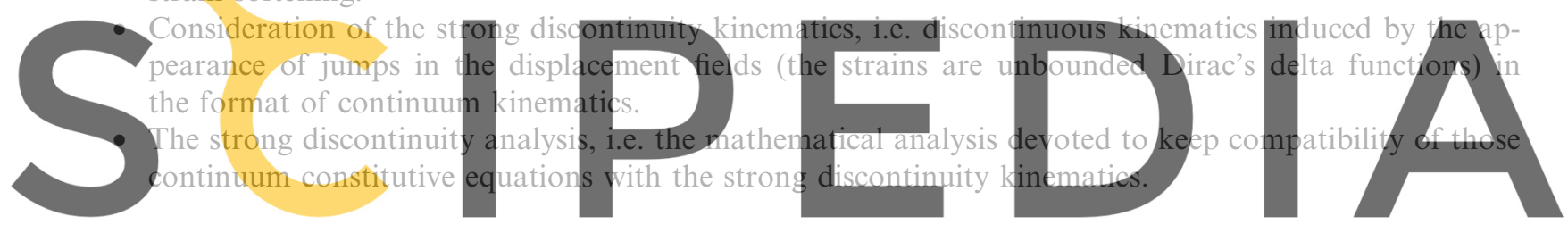

On that basis it is shown that every continuum constitutive model induces, in a consistent manner, a

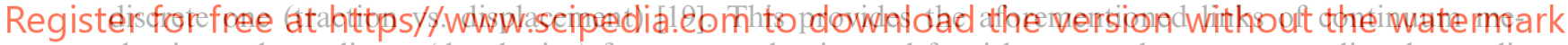
chanics and non-linear (decohesive) fracture mechanics and furnishes procedures to generalize the application of standard constitutive models beyond the continuum range up to reach typical fracture mechanics applications. Examples of the analytical derivation of such discrete models are given in the remaining of this paper for $1 \mathrm{D}$ and $2 \mathrm{D}$ cases on the basis of standard isotropic continuum damage models. Some numerical applications using finite element procedures are also provided.

\section{Motivation: 1D example}

Let us consider the 1D continuum isotropic damage model defined through the following ingredients:

Free energy $\quad \psi(\varepsilon, r)=[1-d(r)] \psi_{0}(\varepsilon) ; \quad \psi_{0}=\frac{1}{2} E \varepsilon^{2}$

Constitutive equation $\sigma=\partial_{\varepsilon} \psi(\varepsilon, r) \Rightarrow \sigma=(1-d) E \varepsilon$

Damage variable $d=1-q(r) / r ; \quad d \in[0,1]$

Evolution law $\dot{r}=\lambda ;\left.\quad r \in\left[\sigma_{u}, \infty\right) \quad r\right|_{t=0}=\sigma_{u}$

Damage criterion $f(\sigma, q) \equiv|\sigma|-q$;

Loading-unloading conditions $\lambda \geqslant 0 ; \quad f \leqslant 0 ; \quad \lambda f=0 ; \underbrace{\lambda \dot{f}=0 \quad \text { for } f=0}_{\text {(consistency) }}$

Hardening rule $\quad \dot{q}=\mathscr{H}(r) \dot{r} \quad\left(\mathscr{H}=q^{\prime}(r) \leqslant 0\right) ;\left.\quad q \in\left[0, \sigma_{u}\right] \quad q\right|_{t=0}=\sigma_{u}$ 
(a)

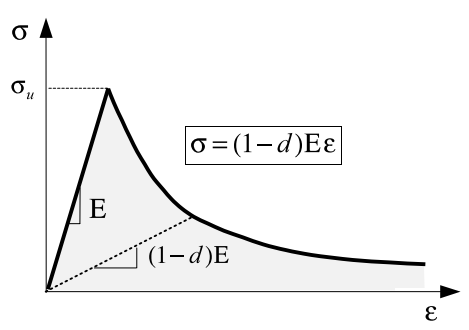

(b)

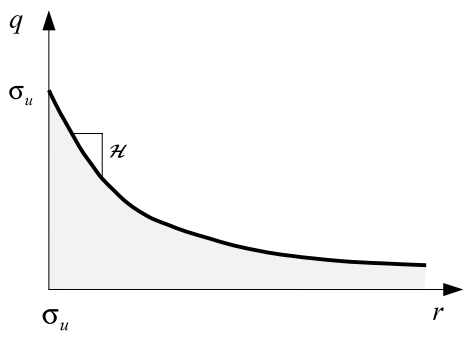

Fig. 1. Uniaxial continuum damage model.

where $\psi$ is the free energy, $\varepsilon$ and $\sigma$ are, respectively, the infinitesimal strain and stress, $r$ is the internal variable and $\sigma_{u}$ is the peak stress (or elastic strength). In addition $\psi_{0}$ is the elastic free energy, $E$ is the Young's modulus and $d$ is the damage variable defined in terms of the hardening/softening variable $q(r)$ whose evolution is ruled by the hardening/softening parameter $\mathscr{H}$. Eq. (1f) describes the loadingunloading conditions where $\lambda \geqslant 0$ is the damage multiplier and $f(\sigma, q)$ defines the damage surface $\left(\partial \mathrm{E}_{\sigma}:=\right.$ $\{\sigma ; f(\sigma, q) \equiv|\sigma|-q=0\})$ which bounds the elastic domain $\left(\mathrm{E}_{\sigma}:=\{\sigma ; f(\sigma, q) \equiv|\sigma|-q<0\}\right)$. Therefore in Eq. (1f) $f=0$ determines that the stress state lies on the damage surface $\partial \mathrm{E}_{\sigma}$. In that case $\lambda=\dot{r}>0$

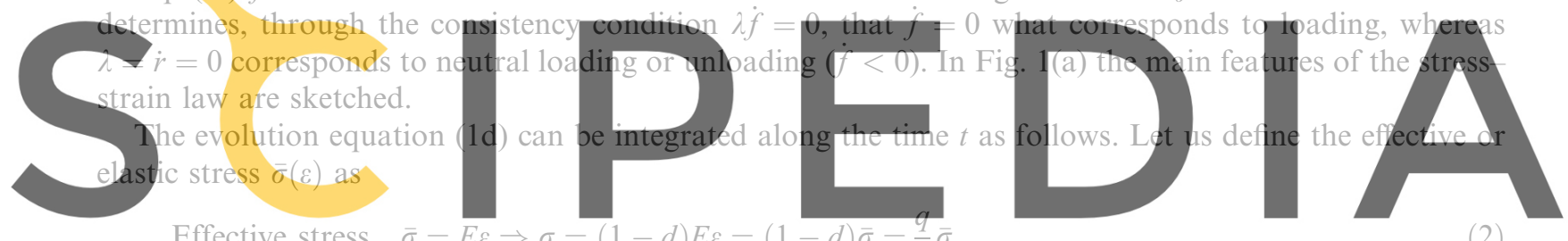

Effective stress $\quad \bar{\sigma}=E \varepsilon \Rightarrow \sigma=(1-d) E \varepsilon=(1-d) \bar{\sigma}=\frac{q}{r} \bar{\sigma}$

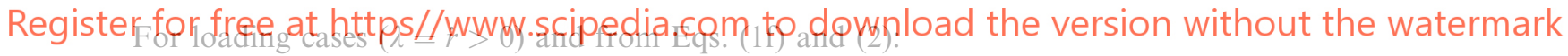

$$
\lambda=\dot{r}>0 \Rightarrow f(\sigma, q) \equiv|\sigma|-q=0 \Rightarrow|\sigma|-q=\underbrace{\frac{q}{r}}_{\geqslant 0}|\bar{\sigma}|-q=0 \Rightarrow r=|\bar{\sigma}|
$$

and since $\lambda=\dot{r} \geqslant 0$ and $\left.r\right|_{t=0}=\sigma_{u}$, from Eqs. (1f) and (1d), Eq. (3) yields:

$$
r(t) \stackrel{\text { not }}{=} r_{t}=\max _{s \in[0, t]}\left[\sigma_{u},|\bar{\sigma}(\varepsilon(s))|\right]
$$

which identifies $r$ as the historical maximum of the effective stress $\bar{\sigma}(\varepsilon)$. Since the hardening/softening law $q(r)$ is assumed to be explicitly known from Eq. (1g), substitution of Eq. (4) into the constitutive Eqs. (1b) and (1c) supplies a closed form expression of the whole damage model.

\subsection{Strong discontinuity kinematics}

Let us consider the 1D bar of Fig. 2 clamped at one end and pulled from the other. Let us denote by $\Omega$ the set of material points of the bar, and let $l$ and $A$ be the bar length and the area of its cross-section, respectively. Let us also consider that a strong discontinuity (namely a jump in the displacement field) takes place at a certain cross-section $S$ of the bar and let us denote by $\Omega / S$ the set of material points of $\Omega$ excluding the ones of $S$ and by $\Omega^{+}$and $\Omega^{-}$those portions of $\Omega / S$ placed in front of and behind of $S$, respectively. Then the discontinuous displacement field can be written as: 


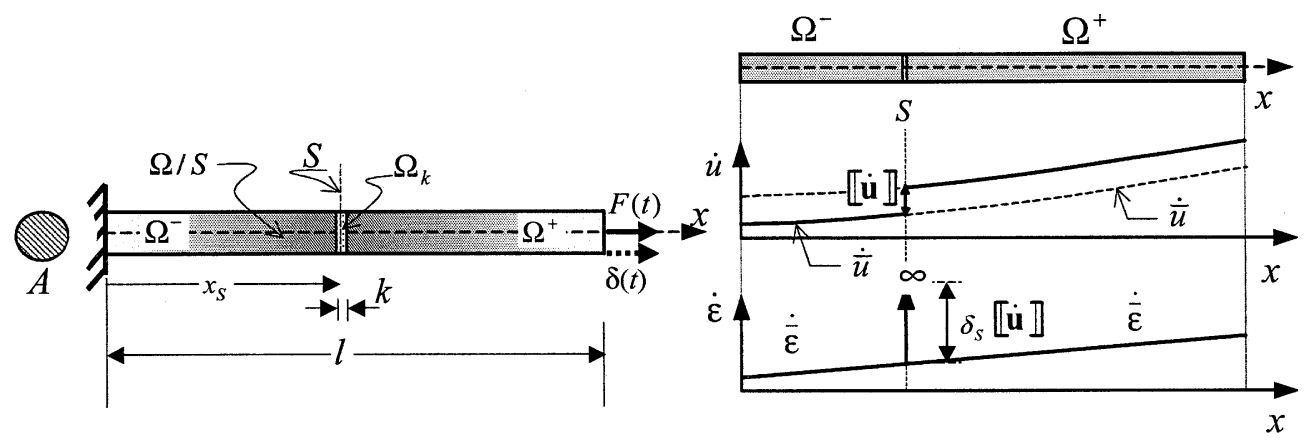

Fig. 2. Strong discontinuity kinematics.

$u(x, t)=\bar{u}(x, t)+H_{S} \llbracket u \rrbracket(x, t)$

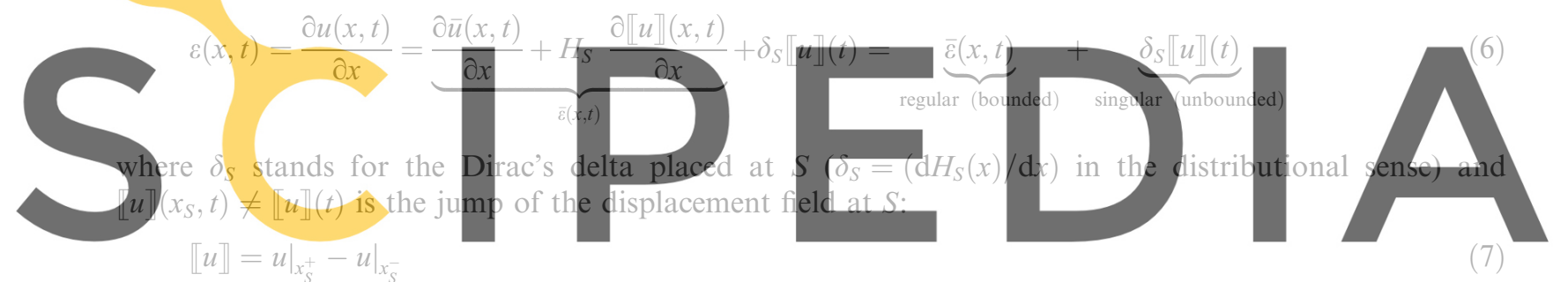

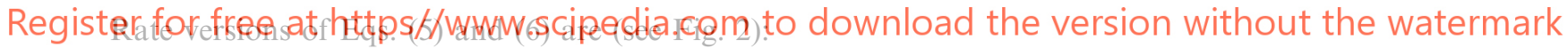

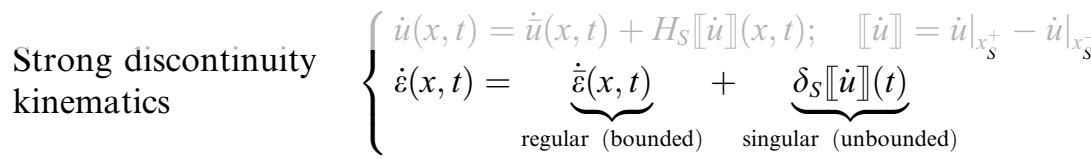

\subsection{Strong discontinuity analysis}

The strong discontinuity analysis aims at making compatible the continuum constitutive model (1) with the unbounded strain field (6). The departure point is the fact that the continuum constitutive model returns bounded stresses (and rate of stresses) for bounded strains (and rate of strains). Therefore, since at $\Omega / S$ the strains $\varepsilon_{\Omega / S}$ are bounded ${ }^{1}\left(\varepsilon_{\Omega / S}=\bar{\varepsilon}=\right.$ bounded and $\dot{\varepsilon}_{\Omega / S}=\dot{\bar{\varepsilon}}=$ bounded), according to Eqs. (6) and (8), then $\sigma_{\Omega / S}$ and $\dot{\sigma}_{\Omega / S}$ are bounded and so are, in virtue of the momentum balance (equilibrium) condition $\left(\sigma_{S}=\sigma_{\Omega / S}\right.$ and $\left.\dot{\sigma}_{S}=\dot{\sigma}_{\Omega / S}\right)$ the stresses $\sigma_{S}$ and $\dot{\sigma}_{S}$ at the discontinuous interface $S$. Consequently, we conclude that both $\sigma_{S}$ and $\dot{\sigma}_{\Omega / S}$ are bounded in spite of the fact that the strains $\varepsilon_{S}$ and $\dot{\varepsilon}_{S}$ can become unbounded due to the presence of the Dirac's delta function $\delta_{S}$ in Eqs. (6) and (8). We shall take advantage of that result in the following.

\footnotetext{
${ }^{1}$ From now on $(\cdot)_{S}$ stands for $\left.(\cdot)(\mathbf{x}, t)\right|_{\mathbf{x} \in S}$ and $(\cdot)_{\Omega / S}$ stands for $\left.(\cdot)(\mathbf{x}, t)\right|_{\mathbf{x} \in \Omega / S}$.
} 
Substitution of Eq. (6) into Eq. (2) yields:

$$
\underbrace{\sigma_{S}}_{\text {bounded }}=\frac{q_{S}}{r_{S}} E\left(\bar{\varepsilon}+\delta_{S} \llbracket u \rrbracket\right)
$$

For the subsequent mathematical treatment, lets us approach the Dirac's delta function $\delta_{S}$ by a $k$-regularized sequence, $\delta_{S}^{k}$, defined as follows: instead of considering the discontinuity surface $S$, we shall consider a discontinuity band $\Omega_{k}$ of bandwidth $k$ (where $k$ is a very small regularization parameter, see Fig. 2) and then:

$$
\delta_{S}^{k}(x) \stackrel{\text { def }}{=} \frac{1}{k} \mu_{S}(x) ; \quad \mu_{S}(x)= \begin{cases}1 & x \in \Omega_{k} \\ 0 & \text { otherwise }\end{cases}
$$

so that, in the distributional sense, $\lim _{k \rightarrow 0} \delta_{S}^{k}=\delta_{S}$. Now, substitution of Eq. (10) into Eq. (9) yields:

$$
\underbrace{\sigma_{S}}_{\text {bounded }}=\frac{q_{S}}{r_{S}} E\left(\bar{\varepsilon}+\frac{1}{k} \llbracket u \rrbracket\right)=\lim _{k \rightarrow 0} \frac{q_{S}}{k r_{S}} E(k \bar{\varepsilon}+\llbracket u \rrbracket)=\left(\lim _{k \rightarrow 0} \frac{1}{k r_{S}}\right) \underbrace{q_{S} E \llbracket u \rrbracket}_{\text {bounded }}
$$

where the bounded character of $q_{S} \in\left[0, \sigma_{u}\right], E$ and $\llbracket u \rrbracket$ is emphasized. Therefore, in order to fulfill the mathematical consistency of Eq. (11) condition $1 / r_{S}=O(k)$ (and, thus, $\lim _{k \rightarrow 0} 1 / k r_{S}=$ bounded in Eq. (11)) has to be guaranteed by introducing an appropriate ingredient in the continuum constitutive model. Let us state this ingredient in terms of the continuum softening parameter $\mathscr{H}$ i.e.
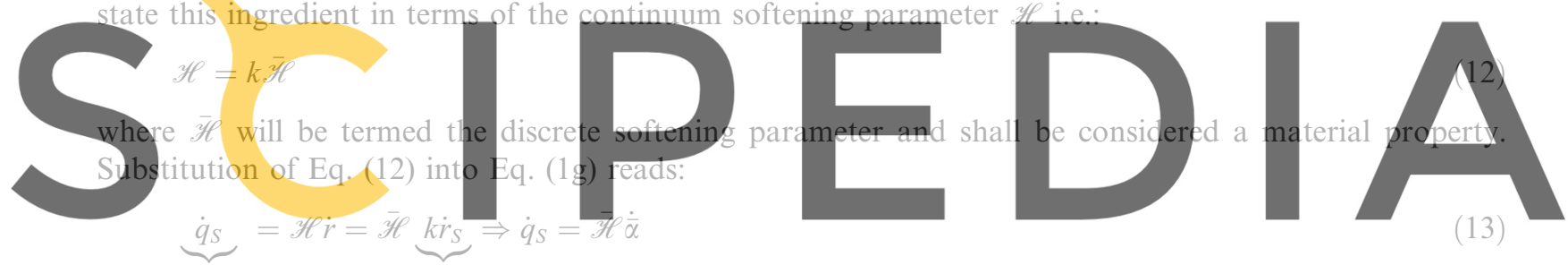

Register for free at https/fwwww.scipedia.com to download the version without the watermark

which defines the discrete internal variable $\bar{\alpha}$ (bounded) as:

Discrete internal variable $\left\{\begin{array}{l}\underbrace{}_{\begin{array}{l}\text { bounded } \\ \left.\bar{\alpha}\right|_{r=\sigma_{u}}\end{array}=0 ; \quad \bar{\alpha} \in[0, \infty\}}=k \dot{r}_{S}\end{array} \Rightarrow \dot{r}_{S}=\frac{1}{k} \dot{\bar{\alpha}} \Rightarrow r_{S}=\sigma_{u}+\frac{1}{k} \bar{\alpha}\right.$

and the discrete hardening/softening variable $\bar{q}(\bar{\alpha})=q_{S}\left(r_{S}\right)$ through the discrete hardening rule:

Discrete hardening rule : $\quad \dot{\bar{q}}(\bar{\alpha})=\overline{\mathscr{H}} \dot{\bar{\alpha}}$ Indeed, the result in Eq. (14) guaranties that $\lim _{k \rightarrow 0} 1 / k r_{S}=\underbrace{1 / \bar{\alpha}}_{\text {bounded }}$ and, consequently, the mathematical
consistency of Eq. (11) which now reads:

$$
\sigma_{S}=\frac{\bar{q}(\bar{\alpha})}{\bar{\alpha}} E \llbracket u \rrbracket
$$

\subsection{Discrete $1 D$ model}

From the continuum density (per unit of volume) of free energy $\psi(\varepsilon, r)$ in Eq. (1a) and the relation $\Omega_{k}=A k$ (see Fig. 2) we can define the discrete (per unit of cross-section surface) free energy, $\varphi$, at the discontinuity interface $S$ as: 
$\varphi \stackrel{\text { def }}{=} \lim _{k \rightarrow 0} \psi\left(\varepsilon_{S}, r_{S}\right) \underbrace{\frac{\Omega_{k}}{A}}_{=k}=\lim _{k \rightarrow 0} k \psi\left(\varepsilon_{S}, r_{S}\right)=\lim _{k \rightarrow 0} \underbrace{\frac{q_{S}}{r_{S}}}_{\left(1-d_{S}\right)} k \frac{1}{2} E \varepsilon^{2}=\lim _{k \rightarrow 0} \underbrace{\frac{q_{S}}{k r_{S}}}_{\frac{q(\tilde{\alpha})}{\alpha}} \frac{1}{2} E(\underbrace{k \varepsilon}_{\llbracket u \rrbracket})^{2}$

Discrete free energy : $\quad \varphi(\llbracket u \rrbracket, \bar{\alpha})=\lim _{k \rightarrow 0} k \psi\left(\varepsilon_{S}, r_{S}\right)=\frac{\bar{q}(\bar{\alpha})}{\bar{\alpha}}\left(\frac{1}{2} E \llbracket u \rrbracket^{2}\right)$

where Eqs. (8), (9) and (14) have been considered. Now, by derivation of Eq. (18), in view of Eq. (16), one obtains:

$$
\partial_{\llbracket u \rrbracket} \varphi(\llbracket u \rrbracket, \bar{\alpha})=\frac{\bar{q}(\bar{\alpha})}{\bar{\alpha}} E \llbracket u \rrbracket=\sigma_{S}
$$

Eq. (19) can now be rewritten as:

Discrete constitutive equation

$$
\begin{aligned}
& \sigma_{S}=(1-\omega) \frac{E}{l} \llbracket u \rrbracket \\
& \omega(\bar{\alpha})=1-\frac{\bar{q}(\bar{\alpha})}{\bar{\alpha}} l
\end{aligned}
$$

Discrete damage variable : $\quad \omega(\bar{\alpha})=1-\frac{\bar{q}(\bar{\alpha})}{\bar{\alpha}} l ; \quad \omega \in(-\infty, 1]$

In Eq. (20) the only role of the bar length $l$ is to keep the dimensionless character of $\omega$. Therefore, the evolution law (1d), taking into account Eq. (14), reads:

$\dot{r}_{S}=\frac{1}{\bar{\alpha}} \dot{\bar{\alpha}}=\lambda \Rightarrow \dot{\bar{\alpha}}=\underbrace{k \lambda}=\bar{\lambda}$

can be written as:

Discrete damage criterion:
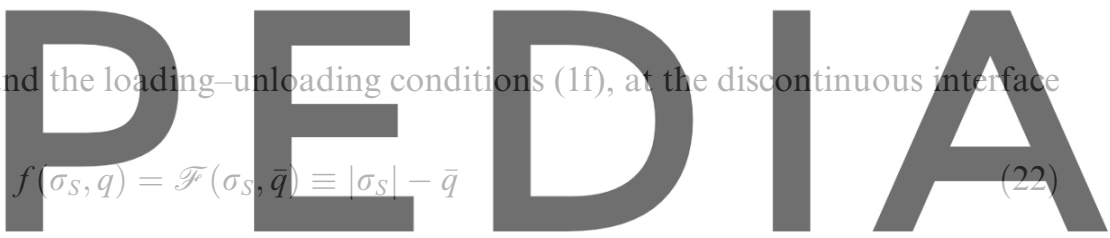

$\bar{\lambda} \geqslant 0 ; \quad \mathscr{F} \leqslant 0 ; \quad \bar{\lambda} \mathscr{F}=0$

(23)

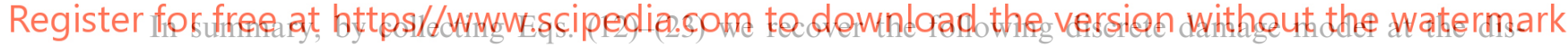
continuous interface $S$ :

Free energy $\underbrace{\varphi(\llbracket u \rrbracket, \bar{\alpha})}_{=\lim _{k \rightarrow 0} k \psi\left(\varepsilon_{S}, r_{S}\right)}=(\bar{q}(\bar{\alpha}) / \bar{\alpha}) \varphi_{0}(\llbracket u \rrbracket) ; \quad \varphi_{0}=\frac{1}{2} E \llbracket u \rrbracket^{2}$

Constitutive equation $\quad \sigma_{S}=\partial_{\llbracket u \rrbracket} \varphi(\llbracket u \rrbracket, \bar{\alpha}) \Rightarrow \sigma_{S}=(1-\omega) \frac{E}{l} \llbracket u \rrbracket$

Damage variable $\omega(\bar{\alpha})=1-\frac{\bar{q}(\bar{\alpha})}{\bar{\alpha}} l ; \quad \omega \in(-\infty, 1]$

Evolution law $\quad \dot{\bar{\alpha}}=\bar{\lambda} ;\left.\quad \bar{\alpha} \in[0, \infty) \quad \bar{\alpha}\right|_{t=0}=0$

Damage criterion $\quad \mathscr{F}\left(\sigma_{S}, \bar{q}\right) \equiv\left|\sigma_{S}\right|-\bar{q}$

Loading-unloading conditions $\quad \bar{\lambda} \geqslant 0 ; \quad \mathscr{F} \leqslant 0 ; \quad \bar{\lambda} \mathscr{F}=0 ; \quad \underbrace{\bar{\lambda} \dot{\mathscr{F}}=0 \text { for } \mathscr{F}=0}_{\text {(consistency) }}$

Hardening rule $\quad \dot{\bar{q}}(\bar{\alpha})=\overline{\mathscr{H}} \dot{\bar{\alpha}} ; \quad \underbrace{\overline{\mathscr{H}}}_{\frac{l}{k} \mathscr{H}}=\bar{q}^{\prime}(\bar{\alpha}) \leqslant 0 ;\left.\quad \bar{q} \in\left[0, \sigma_{u}\right] \quad \bar{q}\right|_{t=0}=\sigma_{u}$

By comparing the discrete damage model (24) with the original continuum damage model (1) we can check that there is a one to one correspondence of the continuum and discrete variables as stated in the following table: 
(a)

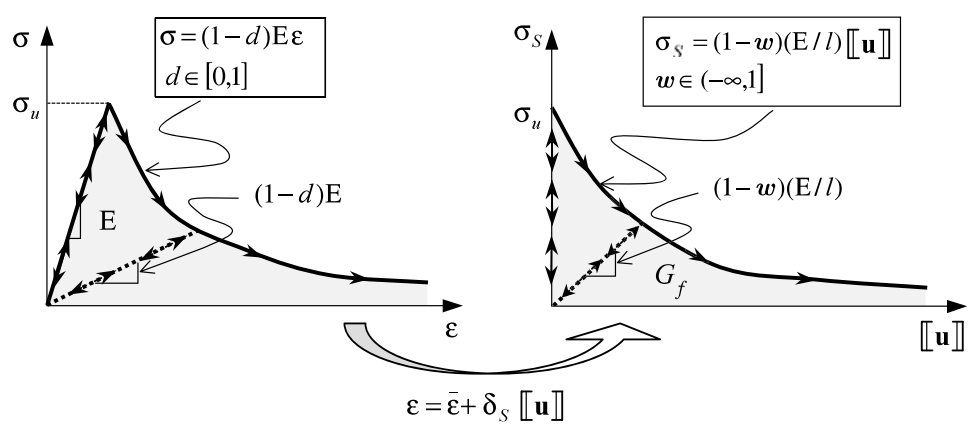

Fig. 3. Continuum and discrete damage models.

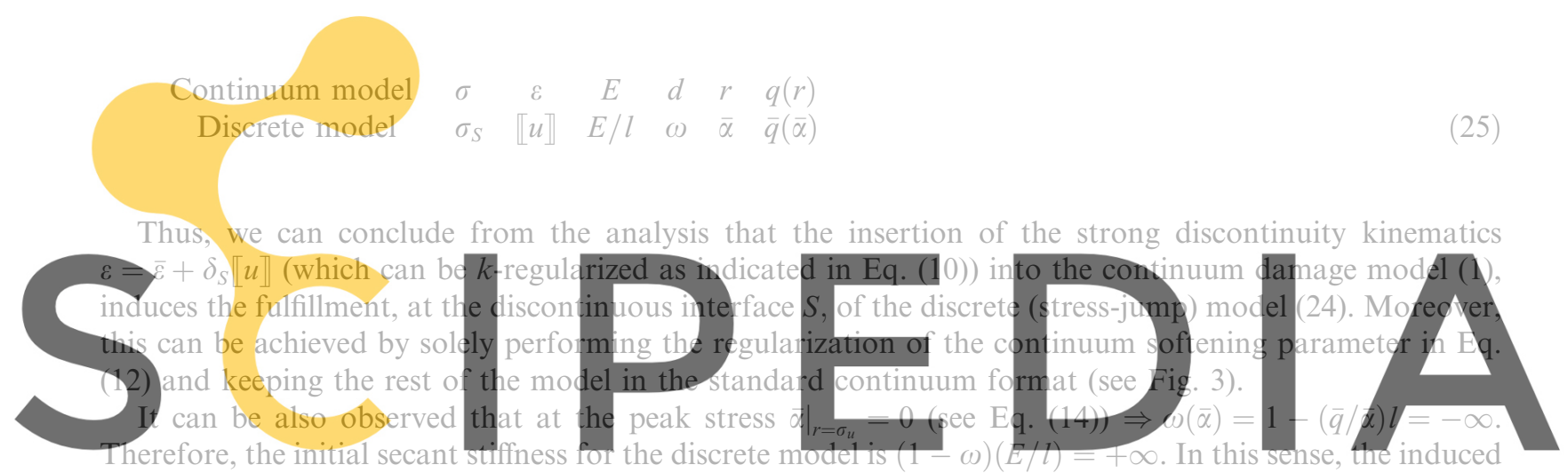

discrete model could be termed as a rigid-damage model.

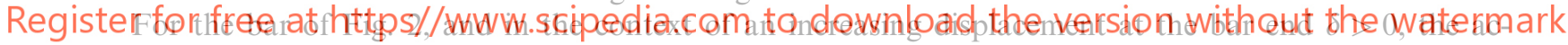

tivation mechanism of such a discrete model is a local bifurcation of the stress-strain fields when the elastic strength $\sigma_{u}$ is reached (which corresponds to a displacement $\delta_{u}=\sigma_{u} l / E$ ). Beyond that point the strong discontinuity kinematics $(8)$ develops $(\llbracket u \rrbracket \neq 0)$ and the constitutive behaviour at the discontinuity interface $S$ is ruled, formally by the non-linear part of the continuum constitutive model (1) (Fig. 3a) but in practice by the discrete constitutive model (24) (Fig. 3b). As far as the continuous part of the bar $\Omega / S$ is concerned, after the bifurcation the constitutive behaviour features a linear elastic unloading, i.e.
$\delta \leqslant \sigma_{u} l / E$
$\delta>\sigma_{u} l / E$
Discontinuous interface $S \quad \sigma=E \bar{\varepsilon} ; \quad \bar{\varepsilon}=\delta / l \quad \sigma=(1-d) E\left(\bar{\varepsilon}+\delta_{S} \llbracket u \rrbracket\right)=(1-\omega) \frac{E}{l} \llbracket u \rrbracket$
Continuous domain $\Omega / S \quad \sigma=E \bar{\varepsilon} ; \quad \bar{\varepsilon}=\delta / l \quad \sigma=E \bar{\varepsilon} ; \quad \dot{\bar{\varepsilon}}=(\dot{\delta}-\llbracket \dot{u} \rrbracket) / l<0)$

\subsection{Fracture mechanics properties: the fracture energy}

It is illustrative to compute the energy consumption for the formation of the discontinuity during the time interval $t \in\left(0, t_{\infty}\right]$, where $t_{\infty}$ stands for the time at which complete decohesion of the crack is achieved $\left(\left.\sigma\right|_{t=t_{\infty}}=0\right)$. In virtue of the theorem of the expended power, and neglecting the kinetic energy (as corresponds to the quasistatic case), the external power input in the bar equals the stress power: 


$$
\begin{aligned}
\mathrm{d} \mathscr{W}(t) & =\underbrace{F \dot{\delta} A}_{\text {External power input }}=\underbrace{\int_{\Omega} \sigma \dot{\varepsilon} \mathrm{d} V}_{\text {Stress power }}=\int_{\Omega} \sigma\left(\dot{\bar{\varepsilon}}+\delta_{S} \llbracket \dot{u} \rrbracket\right) \mathrm{d} V=\int_{\Omega / S} \sigma \underbrace{\dot{\bar{\varepsilon}}}_{\dot{\sigma} / E} \mathrm{~d} V+\int_{S} \sigma \llbracket \dot{u} \rrbracket \mathrm{d} S \\
& =A \int_{0}^{l} \frac{\mathrm{d}}{\mathrm{d} t}\left(\frac{\sigma^{2}}{2 E}\right) \mathrm{d} x+\sigma \llbracket \dot{u} \rrbracket A
\end{aligned}
$$

where the strong discontinuity kinematics (8), the Dirac's delta property $\int_{\Omega} \delta_{S}(\cdot) \mathrm{d} V=\int_{S}(\cdot) \mathrm{d} S$ and the elastic constitutive behaviour at $\Omega / S(\dot{\sigma}=E \dot{\bar{\varepsilon}})$ have been considered. Now, by integration along time, the total mechanical work $\mathscr{W}$ can be computed as:

$$
\begin{aligned}
& \mathscr{W}=\int_{0}^{t_{\infty}} \mathrm{d} \mathscr{W}(t) \mathrm{d} t=\int_{0}^{t_{\infty}}\left(A \int_{0}^{l} \frac{\mathrm{d}}{\mathrm{d} t}\left(\frac{\sigma^{2}}{2 E}\right) \mathrm{d} x+\sigma \llbracket \dot{u} \rrbracket A\right) \mathrm{d} t \\
& =A \int_{0}^{t}\left[\int_{0}^{t_{\infty}} \frac{\mathrm{d}}{\mathrm{d} t}\left(\frac{\sigma^{2}}{2 E}\right) \mathrm{d} t\right] \mathrm{d} x+A \int_{0}^{t_{\infty}} \sigma \llbracket i \bar{u} \rrbracket \mathrm{d} t=A \int_{0}^{l} \underbrace{\left[\frac{\sigma^{2}}{2 E}\right]_{t=0}^{t=t_{\infty}}}_{=0-0} \mathrm{~d} x+A \int_{0}^{t_{\infty}} \sigma \llbracket \dot{u} \rrbracket \mathrm{d} t \\
& =A \underbrace{\int_{0}^{t_{\infty}} \sigma \llbracket \dot{u} \rrbracket \mathrm{d} t}_{G_{\mathrm{f}}}=A G_{\mathrm{f}}
\end{aligned}
$$

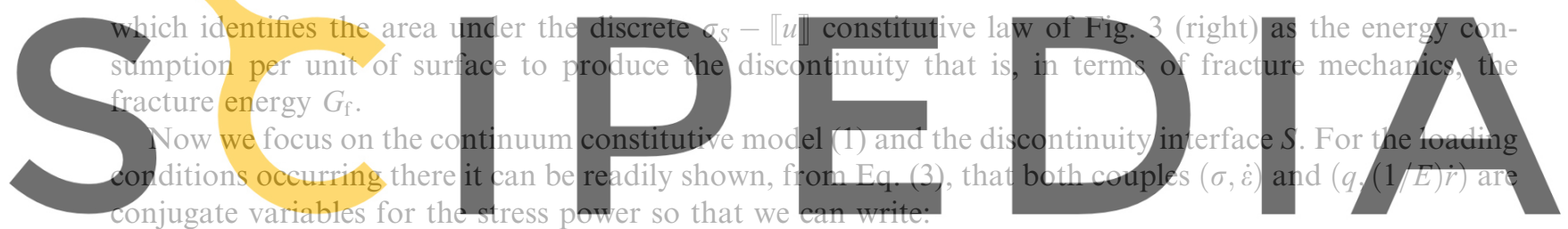

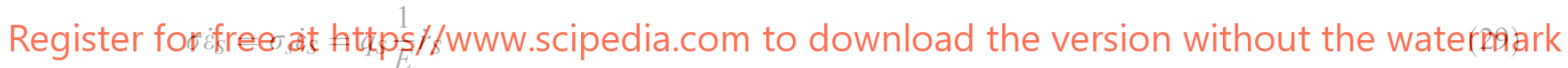

Now, substitution of the $k$-regularized strong-discontinuity kinematics $(\varepsilon=\bar{\varepsilon}+(1 / k) \llbracket u \rrbracket)$ and the regularized hardening softening law from Eq. (13) $\dot{q}_{S}=\overline{\mathscr{H}} k \dot{r}_{S}$ into Eq. (29) yields:

$$
\sigma \dot{\varepsilon}_{S}=\sigma\left(\dot{\bar{\varepsilon}}+\frac{1}{k} \llbracket \dot{u} \rrbracket\right)=q_{S} \frac{1}{E} \dot{r}_{S}=\frac{1}{k E \mathscr{H}} q_{S} \dot{q}_{S}=\frac{1}{k E \mathscr{H}} \frac{\mathrm{d}}{\mathrm{d} t}\left(\frac{1}{2} q_{S}^{2}\right) \Rightarrow \lim _{k \rightarrow 0} k \sigma \dot{\varepsilon}_{S}=\sigma \llbracket \dot{u} \rrbracket=\frac{1}{E \mathscr{H} \mathscr{d}} \frac{\mathrm{d}}{\mathrm{d} t}\left(\frac{1}{2} q_{S}^{2}\right)
$$

Therefore the fracture energy of Eq. (28) can be computed as:

$$
G_{\mathrm{f}}=\int_{0}^{t_{\infty}} \sigma \llbracket \dot{u} \rrbracket \mathrm{d} t=\int_{0}^{t_{\infty}} \frac{1}{E \mathscr{H}} \frac{\mathrm{d}}{\mathrm{d} t}\left(\frac{1}{2} q^{2}\right) \mathrm{d} t=\frac{1}{E \mathscr{H}}\left[\frac{1}{2} q^{2}\right]_{q=q_{0}=\sigma_{u}}^{q=q_{\infty}=0}=-\frac{1}{2} \frac{\sigma_{u}^{2}}{E \mathscr{H}} \Rightarrow \overline{\mathscr{H}}=-\frac{1}{2} \frac{\sigma_{u}^{2}}{E G_{\mathrm{f}}}
$$

where, for the sake of simplicity, a constant value for $\overline{\mathscr{H}}$ has been considered (linear softening). Eq. (31) states the material property character of the discrete softening parameter $\overline{\mathscr{H}}$ which can be then characterized in terms of the fracture mechanics properties $\sigma_{u}, E$, and $G_{\mathrm{f}}$.

\section{Extension to general 2D and 3D problems}

The approach outlined above can be readily extended to the general multidimensional case as follows in next sections. 
a)

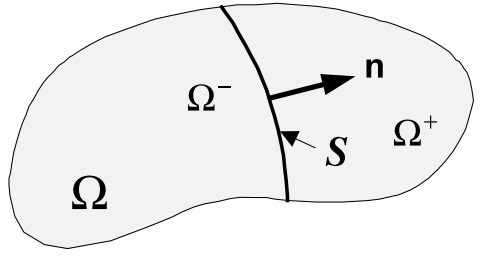

b)

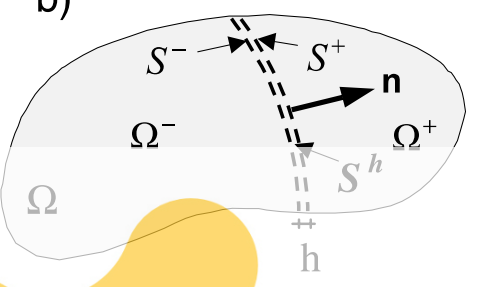

c)

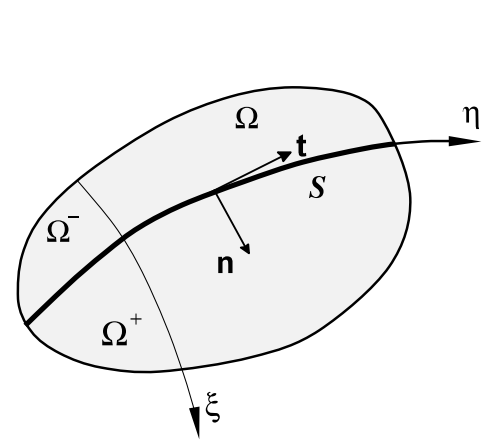

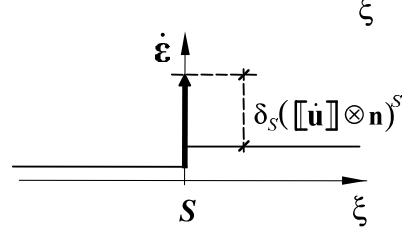

3.1. Kinematics: weak and strong discontinuities

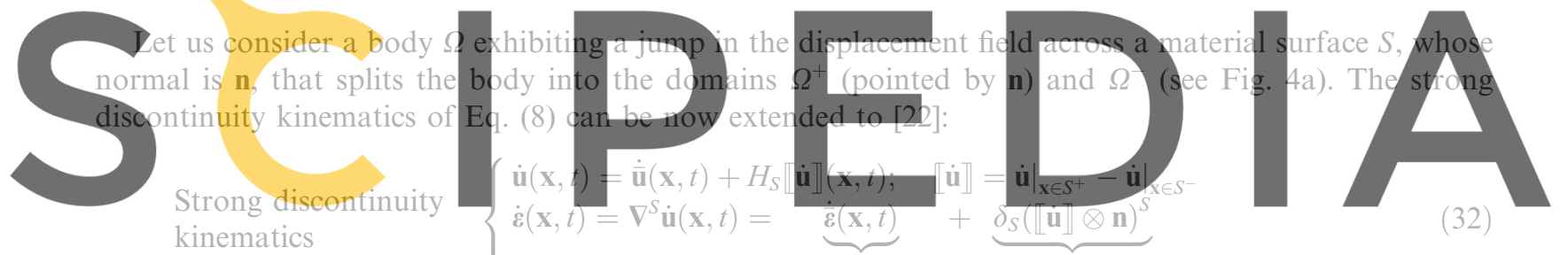

Register for free at https//www.scipedia.com to dow (winto ad the versiont without the watermark

where $H_{S}(\mathbf{x})$ is the step function placed in $S\left(H_{S}(\mathbf{x})=0\right.$ for $\mathbf{x} \in \Omega^{-}$and $H_{S}(\mathbf{x})=1$ otherwise) and $\delta_{S}$ stands for the Dirac's delta function emerging from the gradient (in the distributional sense) of $H_{S}(\mathbf{x})\left(\nabla H_{S}(\mathbf{x})=\right.$ $\left.\delta_{S} \mathbf{n}(\mathbf{x})\right)$.

For the subsequent mathematical analysis it is convenient to consider again a regularized version of the kinematics in Eq. (32) by defining a discontinuity band $S^{h}$ (of bandwidth $h$ ) containing the discontinuity surface $S$ (see Fig. 5) and approaching the Dirac's delta function by the regularized Dirac delta function defined as:

$$
\delta_{S}^{h}(\mathbf{x})=\frac{1}{h} \mu_{S}^{h}(\mathbf{x}) ; \quad \mu_{S}^{h}(\mathbf{x})= \begin{cases}1 & \forall \mathbf{x} \in S^{h} \\ 0 & \forall \mathbf{x} \in \Omega / S^{h}\end{cases}
$$

where $\mu_{S}^{h}(\mathbf{x})$ is a collocation function on $S^{h}$ and $\Omega / S^{h}$ stands for the set of points of $\Omega$ not belonging to $S^{h}$. The regularized version of the rate of strain field in Eq. (32) now reads:

$$
\dot{\boldsymbol{\varepsilon}}(\mathbf{x}, t)=\nabla^{S} \dot{\mathbf{u}}(\mathbf{x}, t)=\underbrace{\dot{\bar{\varepsilon}}(\mathbf{x}, t)}_{\text {regular (bounded) }}+\underbrace{\delta_{S \rightarrow 0)}^{h}(\llbracket \dot{\mathbf{u}} \rrbracket \otimes \mathbf{n})^{S}}_{\text {singular (unbounded when }}
$$

The kinematics of Eq. (34) allows to introduce a new ingredient, the weak discontinuity concept, which appears to be necessary for modelling purposes. We shall consider in Section 3.4 the onset of the discontinuity to originate from a local bifurcation process (discontinuous bifurcation) occurring at time $t_{\mathrm{B}}$ at which the strain fields localizes inside a band $S^{h}$ according to the kinematics of Eq. (34). In the most general 


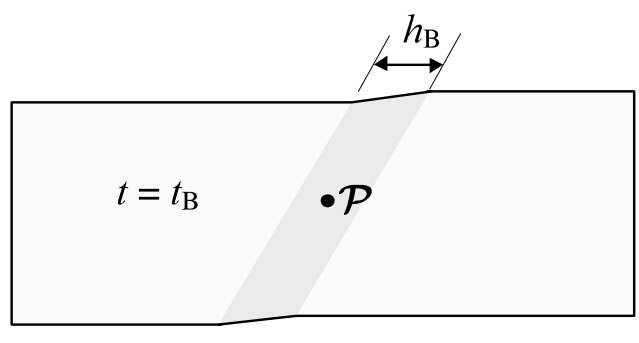

(a)

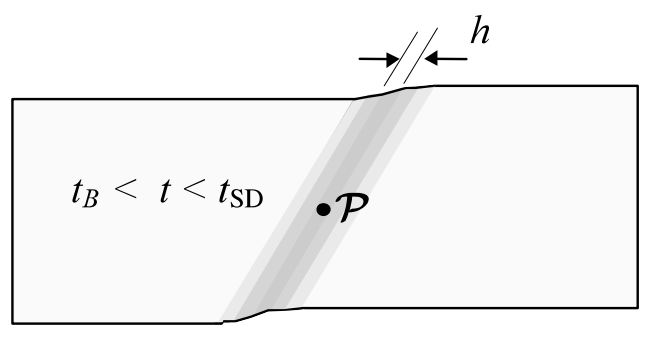

(b)

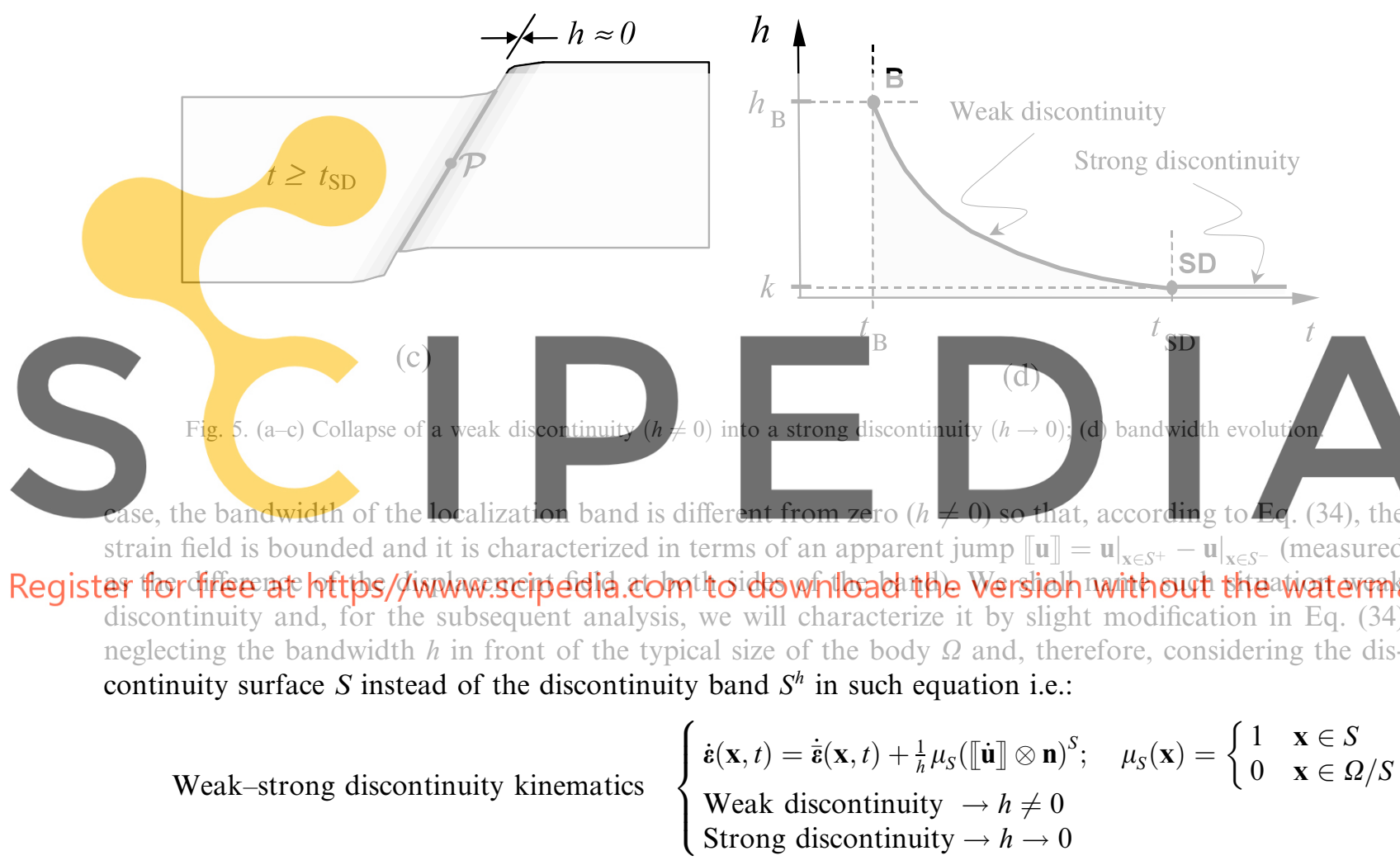

In this context, the process of formation of a strong discontinuity at a material point $\mathscr{P}$ of the body, can be modelled as a weak discontinuity that collapses into a strong discontinuity at a certain time of the deformation process (see Fig. 5). At time $t_{\mathrm{B}}$ (from now on the bifurcation time) the stress-strain field bifurcates, according to the kinematics of Eq. (35), with $\llbracket \dot{\mathbf{u}} \rrbracket \neq 0$, resulting in a localization band of bandwidth $h_{\mathrm{B}}$ (see Fig. 5(a) which characterizes the onset of a weak discontinuity. At subsequent times the bandwidth decreases ruled by a certain (material property) bandwidth evolution law (see Fig. 5(d)) up to reach a null value (for computational purposes, a very small parameter $k$ ) ${ }^{2}$ at time $t_{\mathrm{SD}}$ (from now on the strong discontinuity time) which characterizes the onset of the strong discontinuity. Therefore, during the

\footnotetext{
${ }^{2}$ This minimum bandwidth value, $k$, should be as small as permitted by the machine precision.
} 


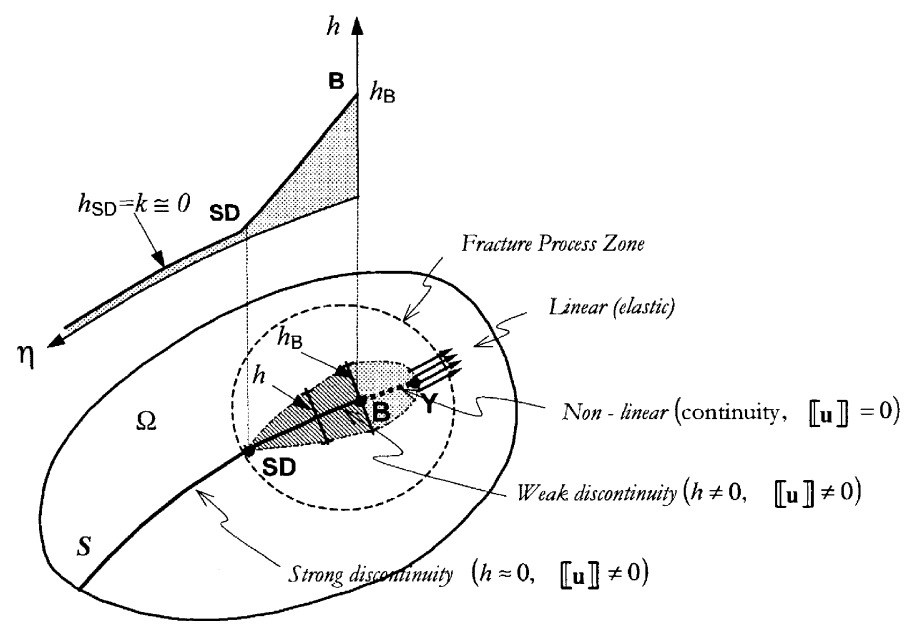

Fig. 6. The fracture process zone modelled by the strong discontinuity approach.

time interval $\left[t_{\mathrm{B}}, t_{\mathrm{SD}}\right]$ a weak discontinuity appears at $\mathscr{P}$ which collapses into a strong discontinuity at time $t=t_{\mathrm{SD}}$. Finally for $t>t_{\mathrm{SD}}$ a full strong discontinuity develops.

Considering now the body level, we can envisage the propagation of the discontinuity as the above process taking place, successively along time, in an increasing set of material points (see Fig. 6). In this set, which defines the propagating discontinuity, we can find the following subsets:

- Material points that have already reached the non-linear (damage, plastic etc.) behaviour but not having bifurcated yet. They define a zone with continuous non-linear behaviour (zone Y-B in Fig. 6).

- Material points that have already bifurcated and thus experiencing a weak discontinuity kinematics, $\llbracket \mathbf{u} \rrbracket \neq 0$, with a non-zero bandwidth (zone B-SD in Fig. 6).

- Material points that have reached the strong discontinuity regime, $h=k \simeq 0$ (zone $S$ in Fig. 6).

According to this, the zone Y-SD in Fig. 6 can be immediately identified as that in which the fracture (strong discontinuity) is processed and, therefore, as the fracture process zone classically considered in nonlinear fracture mechanics $[3,4]$.

\subsection{An isotropic damage model}

The described methodology can be applied to any non-linear constitutive equation equipped with strain softening behaviour. For the sake of simplicity we shall consider here the extension of the one-dimensional isotropic damage model (1) to the general three-dimensional case:

Free energy $\psi(\boldsymbol{\varepsilon}, r)=[1-d(r)] \psi_{0}(\boldsymbol{\varepsilon}) ; \quad \psi_{0}=\frac{1}{2} \boldsymbol{\varepsilon}: \mathbf{C}: \boldsymbol{\varepsilon}$

Constitutive equation $\quad \boldsymbol{\sigma}=\partial_{\varepsilon} \psi(\boldsymbol{\varepsilon}, r) \Rightarrow \boldsymbol{\sigma}=(1-d) \mathbf{C}: \boldsymbol{\varepsilon}$

Damage variable $d=1-q(r) / r ; \quad d \in[0,1]$

Evolution law $\quad \dot{r}=\lambda ; \quad r \in\left[r_{0}, \infty\right) \quad r_{0}=\left.r\right|_{t=0}=\sigma_{u} / \sqrt{E}$

Damage criterion $f(\boldsymbol{\sigma}, q) \equiv \tau_{\sigma}-q ; \quad \tau_{\sigma}=\|\boldsymbol{\sigma}\|_{\mathbf{C}^{-1}}=\sqrt{\boldsymbol{\sigma}: \mathbf{C}^{-1}: \boldsymbol{\sigma}}$ 
Loading-unloading conditions $f \leqslant 0 ; \quad \lambda \geqslant 0 ; \quad \lambda f=0 ; \quad \underbrace{\lambda \dot{f}=0 \text { for } f=0}_{\text {(consistency) }}$

Hardening rule $\dot{q}=\mathscr{H}(r) \dot{r} ; \quad\left(\mathscr{H}=q^{\prime}(r) \leqslant 0\right) ;\left.\quad q \in\left[0, r_{0}\right] \quad q\right|_{t=0}=r_{0}$

In addition to the variables already described in section 2, in Eq. (36a) $\mathbf{C}$ is the elastic fourth order constitutive tensor defined by $\mathbf{C}=\hat{\lambda} \mathbf{1} \otimes \mathbf{1}+2 \mu \mathbf{I}$ (1 and $\mathbf{I}$ are the second and fourth order unit tensors, respectively, and $\hat{\lambda}$ and $\mu$ the Lamé parameters). In Eq. (36e), $\mathbf{C}^{-1}$ defines a metric in the stress space ${ }^{3}$ such that the damage surface $\partial \mathrm{E}_{\sigma}:=\left\{\sigma ; f(\boldsymbol{\sigma}, q) \equiv \tau_{\sigma}-q=\|\boldsymbol{\sigma}\|_{\mathbf{C}^{-1}}-q=0\right\}$ is an ellipsoid in the principal stress space [8].

By defining the equivalent stresses $\overline{\boldsymbol{\sigma}}=\mathbf{C}: \boldsymbol{\varepsilon}$ and the strain based norm $\tau_{\varepsilon}=\|\overline{\boldsymbol{\sigma}}\|_{\mathbf{C}^{-1}}=\sqrt{\overline{\boldsymbol{\sigma}}: \mathbf{C}^{-1}: \overline{\boldsymbol{\sigma}}}=$ $(1 /(1-d)) \tau_{\sigma}$, and following the same reasoning than for the one dimensional case of Section 2 , the model of Eq. (36a)-(36g) can be integrated in closed form, leading to:

$$
r(t) \stackrel{\text { not }}{=} r_{t}=\max _{s \in[0, t]}\left[r_{0}, \tau_{\varepsilon}(\boldsymbol{\varepsilon}(s))\right]
$$

and the tangent (in terms of rates) constitutive equation for loading cases results:

$$
\left\{\begin{array}{l}
\dot{\boldsymbol{\sigma}}=\mathbf{C}^{d}: \dot{\boldsymbol{\varepsilon}} \\
\mathbf{C}^{d}=(1-d) \mathbf{C}-\frac{q-H r}{q^{2} r} \boldsymbol{\sigma} \otimes \boldsymbol{\sigma}
\end{array}\right.
$$

where $\mathbf{C}^{d}$ is the tangent constitutive operator.

\subsection{Strong discontinuity analysis}

As commented above the goal of the strong discontinuity analysis is to extract those features that make the constitutive continuum model (36) compatible with the unbounded strain field (35). The key point for that analysis is to realize that the local form of the linear momentum balance principle imposes the traction, $\mathscr{T}=\boldsymbol{\sigma} \cdot \mathbf{n}$, to be continuous across the discontinuity interface $S$. This traction continuity condition reads:

$$
\mathscr{T}(\mathbf{x}, \mathbf{t})=\boldsymbol{\sigma}_{\Omega / S} \cdot \mathbf{n}=\boldsymbol{\sigma}_{S} \cdot \mathbf{n} \quad \forall \mathbf{x} \in S \quad \forall t \in[0, T]
$$

Since the strains $\varepsilon_{\Omega / S}=\overline{\boldsymbol{\varepsilon}}$, at the continuous neighbourhood of $S$, are bounded according to Eq. (34), and since the continuum constitutive model returns bounded stresses for bounded strains (or rates of strains) we can conclude that $\boldsymbol{\sigma}_{\Omega / S}\left(\boldsymbol{\varepsilon}_{\Omega / S}\right)$ is bounded and so is $\mathscr{T}=\boldsymbol{\sigma}_{\Omega / S} \cdot \mathbf{n}$. Consequently, from Eq. (39), $\boldsymbol{\sigma}_{S} \cdot \mathbf{n}$ has to be also bounded and so must be the whole tensor $\sigma_{S}$ even for $t \geqslant t_{\mathrm{SD}}$ (the strong discontinuity regime) when $h \rightarrow 0$ and the strains $\varepsilon_{S}$ are unbounded according to Eq. (34) (see Ref. [19] for further details). A subsequent analysis shows that also $q_{S}, \dot{\sigma}_{S}$ and $\dot{q}_{S}$ are bounded at the strong discontinuity regime. Based on those facts a similar analysis to the one of Sections 2.2 and 2.3 can be performed. The main results from this analysis are:

(1) The softening regularization condition:

$$
\mathscr{H}=k \overline{\mathscr{H}} \text { for } t \geqslant t_{\mathrm{SD}}
$$

in terms of the continuum softening parameter $\mathscr{H}$ and the discrete softening parameter $\overline{\mathscr{H}}$, as a necessary condition for the aforementioned compatibility.

(2) The following discrete damage constitutive model emerging from the traction continuity condition (39) (more details on its derivation can be found in Ref. [19]):

\footnotetext{
${ }^{3}$ For uniaxial stress states it results $\tau_{\sigma}=\|\boldsymbol{\sigma}\|_{\mathbf{C}^{-1}}=|\sigma| / \sqrt{E}$.
} 
$\begin{array}{ll}\text { Free energy } & \varphi(\Delta \llbracket \mathbf{u} \rrbracket, \Delta \bar{\alpha})=\frac{\bar{q}(\Delta \bar{\alpha})}{\Delta \bar{\alpha}} \varphi_{0}(\Delta \llbracket \mathbf{u} \rrbracket) \\ & \varphi(\Delta \llbracket \mathbf{u} \rrbracket, \Delta \bar{\alpha}) \stackrel{\operatorname{def}}{=} \lim _{h \rightarrow 0} h \psi\left(\varepsilon_{S}, r_{S}\right)\end{array}\left\{\begin{array}{l}\varphi_{0}=\frac{1}{2} \Delta \llbracket \mathbf{u} \rrbracket \cdot \mathbf{Q}^{e} \cdot \Delta \llbracket \mathbf{u} \rrbracket \\ \mathbf{Q}^{e}(\mathbf{n})=\mathbf{n} \cdot \mathbf{C} \cdot \mathbf{n}\end{array}\right.$

Constitutive equation $\quad \mathscr{T}=\partial_{\llbracket \mathbf{u} \rrbracket} \varphi(\Delta \llbracket \mathbf{u} \rrbracket, \Delta \bar{\alpha}) \quad \mathscr{T}=(1-\omega) \mathbf{Q}^{e} \cdot \Delta \llbracket \mathbf{u} \rrbracket / l$

Damage variable $\omega(\Delta \bar{\alpha})=1-\frac{\bar{q}(\Delta \bar{\alpha})}{\Delta \bar{\alpha}} l \quad \omega \in(-\infty, 1]$

Evolution law $\quad \dot{\bar{\alpha}}=\bar{\lambda} \quad\left(\dot{\bar{\alpha}} \stackrel{\text { def }}{=} \lim _{h \rightarrow 0} \dot{r}\right) \quad\left\{\begin{array}{l}\Delta \bar{\alpha} \in[0, \infty) \\ \left(\left.\Delta \bar{\alpha} \stackrel{\text { def }}{=} \bar{\alpha}\right|_{t}-\left.\bar{\alpha}\right|_{t=t_{\mathrm{SD}}}\right)\end{array}\right.$

Damage criterion $\quad \mathscr{F}(\mathscr{T}, \bar{q}) \equiv \tau_{\mathscr{T}}-\bar{q} \quad \tau_{\mathscr{T}}=\|\mathscr{T}\|_{\left[\mathbf{Q}^{e}\right]^{-1}}=\sqrt{\mathscr{T} \cdot\left[\mathbf{Q}^{e}\right]^{-1} \cdot \mathscr{T}}$

Loading-unloading conditions $f \leqslant 0 ; \lambda \geqslant 0 ; \quad \lambda f=0 \underbrace{\bar{\lambda} \dot{\mathscr{F}}=0 \quad \text { for } \mathscr{F}=0}_{\text {(consistency) }}$

$\begin{array}{lll}\text { Hardening rule } & \dot{\bar{q}}(\Delta \bar{\alpha})=\overline{\mathscr{H}} \dot{\bar{\alpha}} ; \overline{\mathscr{H}}=\bar{q}^{\prime}(\Delta \bar{\alpha}) \leqslant 0 & \bar{q} \in\left[0, q_{\mathrm{SD}}\right] \\ & q_{\mathrm{SD}}=\left.q\right|_{t=t_{\mathrm{SD}}}\end{array}$

where, $\Delta \llbracket \mathbf{u} \rrbracket=\llbracket \mathbf{u} \rrbracket_{t}-\llbracket \mathbf{u} \rrbracket_{t_{\mathrm{SD}}}$ stands for the incremental jump from the onset of the strong discontinuity. ${ }^{4}$ In Eq. (41a), $\mathbf{Q}^{e}(\mathbf{n})=\mathbf{n} \cdot \mathbf{C} \cdot \mathbf{n}$ is the so called acoustic tensor which can be proved to be non-singular $\left(\operatorname{det} \mathbf{Q}^{e}(\mathbf{n})>0\right)[34]$.

(3) The strong discontinuity conditions. Unlike the 1D analysis of Section 2, in general 2D-3D cases, some restrictions on the structure of stress field $\sigma_{S}$ during the strong discontinuity regime $\left(t \geqslant t_{\mathrm{SD}}\right)$ appear from the strong discontinuity analysis in addition to the discrete constitutive model (41). Those restrictions, which can be formally written as $\mathbf{R}\left(\boldsymbol{\sigma}_{S}\right)=\mathbf{0}$, have to be fulfilled all along the strong discontinuity regime and, in particular, at the strong discontinuity time $t_{\mathrm{SD}}$. For the damage models considered here these conditions can be shown to be [19]:

$$
\text { Strong discontinuity conditions } \quad \mathbf{R}\left(\boldsymbol{\sigma}_{S}\right) \equiv\left\{\begin{array}{l}
\bar{\varepsilon}_{22_{S}}=\bar{\varepsilon}_{23_{S}}=\bar{\varepsilon}_{33_{S}}=0 \\
\Rightarrow\left[\mathbf{C}^{-1}: \boldsymbol{\sigma}_{S}\right]_{22}=\left[\mathbf{C}^{-1}: \boldsymbol{\sigma}_{S}\right]_{23}=\left[\mathbf{C}^{-1}: \boldsymbol{\sigma}_{S}\right]_{33}=0
\end{array}\right.
$$

where $\bar{\varepsilon}=\mathbf{C}^{-1}: \boldsymbol{\sigma}$ stands for the effective strains and subscripts $(\cdot)_{i j}$ refer to tensorial components in a local orthogonal coordinate system defined by the unit vectors $\left\{\mathbf{n} \equiv \hat{\mathbf{e}}_{1}, \hat{\mathbf{e}}_{2}, \hat{\mathbf{e}}_{3}\right\}$ at every point of the discontinuous interface $S$.

Remark 1. Again, the following one to one correspondence of the continuum constitutive model (36) and the induced discrete one (41) can be found:

\footnotetext{
${ }^{4}$ Notice that the possibility $\llbracket \mathbf{u} \rrbracket_{t_{\mathrm{SD}}} \neq 0$ is considered here. This corresponds to the case, tackled in Section 3.1, that a displacement jump $\llbracket \mathbf{u} \rrbracket$ develops during the weak discontinuity regime $\left(t \in\left[t_{\mathrm{B}}, t_{\mathrm{SD}}\right]\right)$ preceding the strong discontinuity.
} 


\begin{tabular}{|c|c|c|}
\hline Continuum model & Discrete model & \\
\hline$\psi(\boldsymbol{\varepsilon}, r)$ & $\varphi(\Delta \llbracket \mathbf{u} \rrbracket, \Delta \bar{\alpha})=\lim _{h \rightarrow 0} h \psi\left(\boldsymbol{\varepsilon}_{S}, r_{S}\right)$ & \\
\hline $\boldsymbol{\varepsilon}$ & $\Delta \llbracket \mathbf{u} \rrbracket$ & \\
\hline $\boldsymbol{\sigma}=\partial_{\varepsilon} \psi(\boldsymbol{\varepsilon}, r)=(1-d) \mathbf{C}: \boldsymbol{\varepsilon}$ & $\begin{array}{c}\mathscr{T}=\partial_{\Delta \llbracket \mathbf{u} \rrbracket} \varphi(\Delta \llbracket \mathbf{u} \rrbracket, \Delta \bar{\alpha})=(1-\omega) \mathbf{Q}^{e} \cdot \Delta \llbracket \mathbf{u} \rrbracket / l \\
\mathbf{Q}^{e}(\mathbf{n})=\mathbf{n} \cdot \mathbf{C} \cdot \mathbf{n}\end{array}$ & \\
\hline$r$ & $\Delta \bar{\alpha}=\lim _{h \rightarrow 0} h r$ & (43) \\
\hline$q(r)$ & $\bar{q}(\Delta \bar{\alpha})$ & \\
\hline$d=1-q / r$ & $\omega=1-l \bar{q} / \Delta \bar{\alpha}$ & \\
\hline $\begin{array}{c}f(\boldsymbol{\sigma}, q)=\tau_{\sigma}-q \\
\tau_{\sigma}=\|\boldsymbol{\sigma}\|_{\boldsymbol{C}^{-1}} \\
\mathscr{H}=\overline{\mathscr{H} h}\end{array}$ & $\begin{array}{c}\mathscr{F}(\mathscr{T}, \bar{q})=\tau_{\mathscr{T}}-\bar{q} \\
\tau_{\mathscr{T}}=\|\mathscr{T}\|_{\left[\mathbf{Q}^{e}\right]^{-1}}\end{array}$ & \\
\hline
\end{tabular}

Remark 2. The constitutive model in Eq. (41a)-(41g) is a discrete damage model relating the traction $\mathscr{T}$ at the discontinuity interface and the displacement jump $\llbracket \mathbf{u} \rrbracket$. Several important features can be noticed here:

(1) The discrete model is automatically induced by the strong discontinuity kinematics (35) and the traction continuity condition (39) on the basis of the continuum model of Eq. (36a)-(36g), and there is no need to derive it explicitly. For modelling purposes the whole simulation is kept in the continuum format.

(2) The discrete model inherits the nature of the parent continuum model. For the present case the damage character of the continuum damage model $(\boldsymbol{\sigma}=(1-d) \mathbf{C}: \boldsymbol{\varepsilon})$ is inherited by the discrete damage model $\left(\mathscr{T}=(1-\omega) \mathbf{Q}^{e} \cdot \Delta \llbracket \mathbf{u} \rrbracket / l\right)$. Moreover, it can be observed that, since $\left.\Delta \bar{\alpha}\right|_{t_{\mathrm{SD}}}=0$ then $\left.\omega\right|_{t_{\mathrm{SD}}}=\infty$ (see Eq. (41c)) and the initial secant discrete constitutive model is $\mathbf{Q}^{s}=(1-\omega) \mathbf{Q}^{e}=-\infty \mathbf{Q}^{e}$ (see Eq. (41b)). Therefore the discrete damage model is a rigid-damage one. Those facts can be proved to happen also with other parent continuum constitutive models (plasticity models for instance), see Ref. [19] for details.

Remark 3. The strong discontinuity conditions (42) clearly preclude, for general cases, the formation of a strong discontinuity directly by discontinuous bifurcation from a continuous stress-strain field. Unless those conditions are fulfilled by that stress field, ${ }^{5}$ the bifurcation must take place under a different kinematics. This suggest the weak discontinuity concept and the variable bandwidth model, outlined in Section 3.1, as appropriate modelling mechanisms to allow such bifurcation in general cases and to progressively induce the strong discontinuity conditions (42).

\subsection{Onset of the discontinuity: bifurcation analysis}

At this point the following questions, with respect to the mechanism of formation and propagation of the discontinuity outlined in Section 3.1, remain to be answered:

- How the bifurcation time $t_{\mathrm{B}}$, signalling the onset of the weak discontinuity at a given material point, is determined?

- What is the procedure to set up the normal $\mathbf{n}$ that appears in the kinematical description of Eq. (35)?

- How the bandwidth $h_{\mathrm{B}}$ at the bifurcation time of Fig. 5(d) is determined?

\footnotetext{
${ }^{5}$ Which only happens for very particular loading conditions.
} 
Those questions can be tackled by resorting to the tools provided by the discontinuous bifurcation analysis [28] which focus on the problem of the bifurcation of the stress-strain fields in the neighbourhood of a given material point $\mathscr{P} \in S$, constrained by the rate form of the traction continuity condition (39):

$$
\llbracket \dot{\mathscr{T}} \rrbracket\left(\mathbf{x}_{\mathscr{P}}, t_{\mathrm{B}}\right)=\llbracket \dot{\boldsymbol{\sigma}} \rrbracket \cdot \mathbf{n}=0
$$

The problem can be stated as follows: find under what conditions the stress-strain fields, continuous in a neighbourhood of $\mathscr{P}\left(\boldsymbol{\sigma}_{S}=\boldsymbol{\sigma}_{\Omega / S}, \boldsymbol{\varepsilon}_{S}=\boldsymbol{\varepsilon}_{\Omega / S}\right)$, bifurcate into discontinuous rate of stress and strain fields (35) such that:

$$
\left\{\begin{array}{l}
\dot{\boldsymbol{\sigma}}_{\Omega / S}\left(\mathbf{x}_{\mathscr{P}}, t_{\mathrm{B}}\right)=\mathbf{C}_{\Omega / S}: \dot{\boldsymbol{\varepsilon}}_{\Omega / S}=\mathbf{C}_{\Omega / S}: \dot{\overline{\boldsymbol{\varepsilon}}} \\
\dot{\boldsymbol{\sigma}}_{S}\left(\mathbf{x}_{\mathscr{P}}, t_{\mathrm{B}}\right)=\mathbf{C}_{S}: \dot{\boldsymbol{\varepsilon}}_{S}=\mathbf{C}_{S}:\left[\overline{\overline{\boldsymbol{\varepsilon}}}+\frac{1}{h}\left(\llbracket \dot{\mathbf{u}} \rrbracket_{t=t_{\mathrm{B}}} \otimes \mathbf{n}\right)^{S}\right]
\end{array}\right.
$$

subjected to the condition (44). In Eq. (45) $\mathbf{C}_{\Omega / S}$ and $\mathbf{C}_{S}$ stand for the tangent constitutive operator at the continuous and discontinuous neighbourhoods, respectively, of $\mathscr{P}$. It can be shown [28] that the most unfavourable case (first bifurcation) corresponds to bifurcation under neutral loading at $\Omega / S\left(\mathbf{C}_{\Omega / S}=\mathbf{C}\right)$ and loading at $S\left(\mathbf{C}_{S}=\mathbf{C}^{d}(\mathscr{H})\right.$, see Eq. (38)). Some algebraic manipulation of Eqs. (44) and (45) leads finally to:

$$
(\underbrace{\mathbf{n} \cdot \mathbf{C}^{d} \cdot \mathbf{n}}_{\mathbf{Q}^{d}}) \cdot \llbracket \dot{\mathbf{u}} \rrbracket_{t=t_{\mathrm{B}}}=\mathbf{Q}^{d} \cdot \llbracket \dot{\mathbf{u}} \rrbracket_{t=t_{\mathrm{B}}}=0
$$

where $\mathbf{Q}^{d}(\mathscr{H}, \mathbf{n})=\mathbf{n} \cdot \mathbf{C}^{d}(\mathscr{H}) \cdot \mathbf{n}$ is termed the localization tensor [31,34]. Since we look for the first time, $t=t_{\mathrm{B}}$, that at point $\mathscr{P}$ occurs that $\llbracket \dot{\mathbf{u}} \rrbracket_{t=t_{\mathrm{B}}}=0,{ }^{6}$ from Eq. (46) the localization tensor has to be singular:

$$
\operatorname{det}\left[\mathbf{Q}^{d}(\mathscr{H}, \mathbf{n})\right]=0
$$

Let us consider the set $\mathscr{G}$ of values of the softening parameter $\mathscr{H}$ fulfilling Eq. (47):

$$
\mathscr{G}:=\left\{\mathscr{H} \in \mathbb{R} \mid \exists \mathbf{n} ; \quad \operatorname{det}\left[\mathbf{Q}^{d}(\mathscr{H}, \mathbf{n})\right]=0\right\}
$$

In the elastic range $\mathbf{Q}^{d}=\mathbf{Q}^{e}$ and it can be shown that $\operatorname{det}\left(\mathbf{Q}^{e}\right)>0[34]$ and, therefore, $\mathscr{G}=\{\emptyset\}$. Beyond the peak stress $\mathscr{G}$ is not empty and, in general, contains more than one value. Therefore a choice is made for the highest value in $\mathscr{G}$ (denoted by $\mathscr{H}_{\text {crit }}$ ), and the corresponding value of $\mathbf{n}$ (denoted by $\mathbf{n}_{\text {crit }}$ ) fulfilling $\operatorname{det}\left[\mathbf{Q}^{d}\left(\mathscr{H}_{\text {crit }}, \mathbf{n}_{\text {crit }}\right)\right]=0$ :

$$
\left\{\begin{array}{l}
\mathscr{H}_{\text {crit }}=\max _{\mathscr{H} \in \mathscr{G}} \mathscr{H} \\
\mathbf{n}_{\text {crit }}=\left\{\mathbf{n} \mid \quad\|\mathbf{n}\|=1 ; \quad \operatorname{det}\left[\mathbf{Q}^{d}\left(\mathscr{H}_{\text {crit }}, \mathbf{n}\right)\right]=0\right\}
\end{array}\right.
$$

The first time that the value of the continuum softening parameter $\mathscr{H}$ (considered a material property) at point $\mathscr{P}$ equals the critical value $\mathscr{H}_{\text {crit }}$ signals the bifurcation time $t_{\mathrm{B}}$ at that point and determines that value of $\mathbf{n}$ :

$$
\mathscr{H}\left(\mathbf{x}_{\mathscr{P}}, t\right)=\mathscr{H}_{\text {crit }}\left(\mathbf{x}_{\mathscr{P}}, t\right) \rightarrow t_{\mathrm{B}}(\mathscr{P})=t \rightarrow \mathbf{n}\left(\mathbf{x}_{\mathscr{P}}, t\right)=\mathbf{n}_{\text {crit }}\left(\mathbf{x}_{\mathscr{P}}, t_{\mathrm{B}}\right) \quad \forall t \geqslant t_{\mathrm{B}}
$$

On the other hand, if we extend the softening regularization condition (40), to the weak discontinuity regime we have, from Eq. (50):

$$
\text { Extended softening regularization condition } \quad \mathscr{H}=h \overline{\mathscr{H}} \quad \text { for } t \geqslant t_{\mathrm{B}} \Rightarrow h_{\mathrm{B}}=\frac{\mathscr{H}_{\mathrm{B}}}{\overline{\mathscr{H}}}=\frac{\mathscr{H}_{\text {crit }}}{\overline{\mathscr{H}}}
$$

which provides the initial value $h_{\mathrm{B}}$ of the bandwidth law of Fig. 5(d).

\footnotetext{
${ }^{6}$ Otherwise there is no bifurcation.
} 


\section{Computational aspects: numerical simulation}

The approach outlined above requires, for numerical simulation purposes via finite elements, the use of specific elements which are able to capture the discontinuous kinematics of Eq. (35). Recent developments on that field lead to the formulation of the so called finite elements with embedded discontinuities $[1,7,11,13,18]$ which, essentially, supplement the standard finite elements (triangular, quadrilateral etc.) with additional internal degrees of freedom whose associated shape functions are discontinuous inside the element and, therefore, match the weak and strong discontinuity kinematics (see Fig. 7).

Using such finite elements some typical drawbacks of standard finite elements when dealing with strain softening problems (mesh size and mesh bias unobjectivity) can be completely removed [18] without the necessity of any remeshing procedure. The strong discontinuity is captured by a set of elements which propagate along the finite element mesh. The locus of the interface $S_{e}$ in such elements (see Fig. 7) defines the discontinuity path representing the actual crack or fracture.

In order to show the capability of the approach, some simulations of typical fracture mechanics problems are presented in next sections. In all the cases the numerical results have been obtained by using the SDA on the basis of the isotropic continuum damage model sketched in Section 3.2. A standard nonlinear finite element code was modified to introduce the aforementioned finite elements with an embedded discontinuity and to regularize the softening law of the continuum damage model according to Eq. (51). The discrete softening parameter $\overline{\mathscr{H}}$ was computed in terms of the fracture energy $G_{\mathrm{f}}$ according to Eq. (31). The continuum softening parameter $\mathscr{H}$ was taken equal to the discrete one $(\mathscr{H}=\overline{\mathscr{H}})$.

\subsection{Notched specimen in plane stress}

Fig. 8(a) shows a notched concrete specimen tested by Kobayashi et al. [12]. The couples of forces $F_{1}$ and $F_{2}$ are progressively applied, as it is indicated, in Fig. 8(b) in order to induce a mixed mode crack which

a)

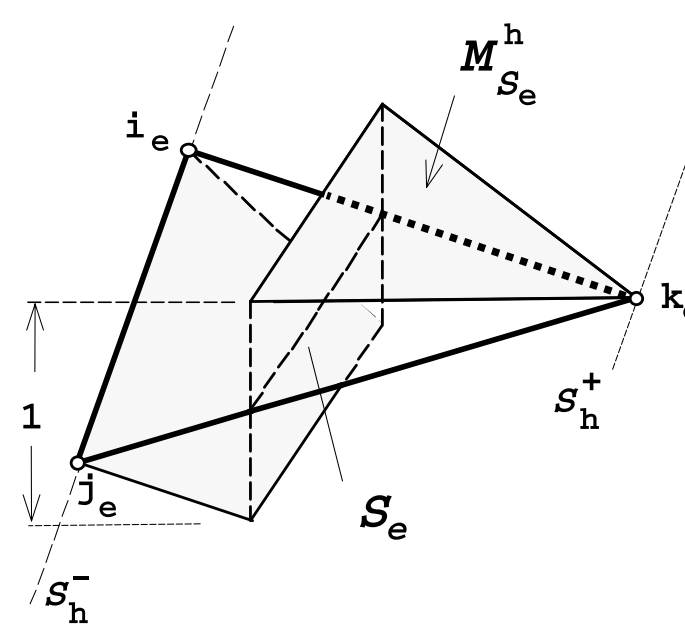

b)

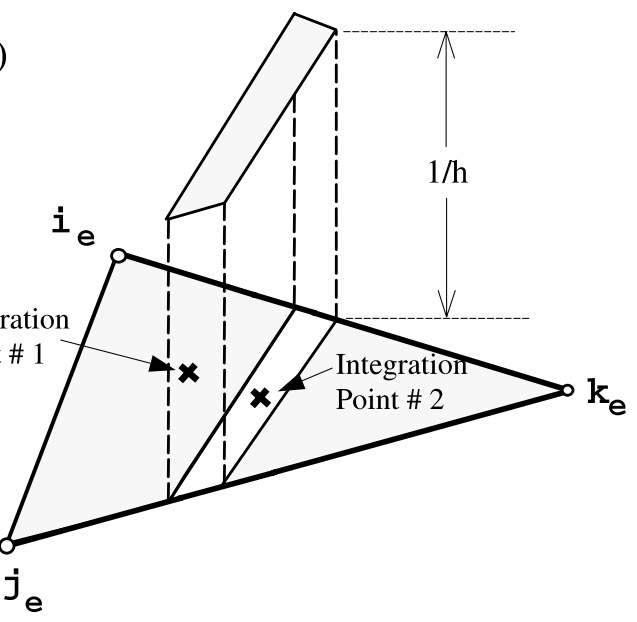

Fig. 7. (a) Discontinuity shape function. (b) Regularized delta-sequence. 

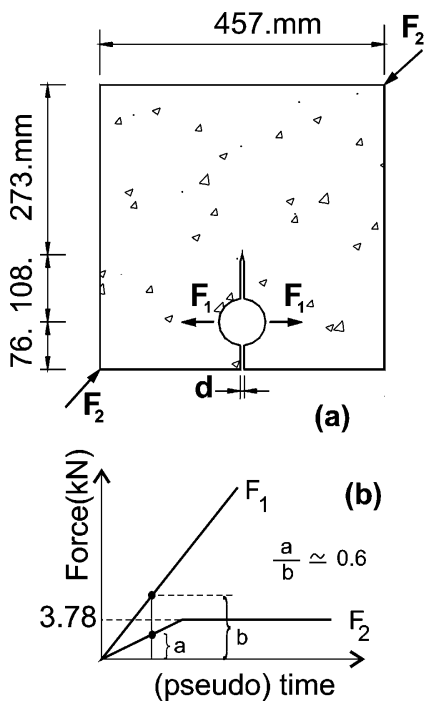

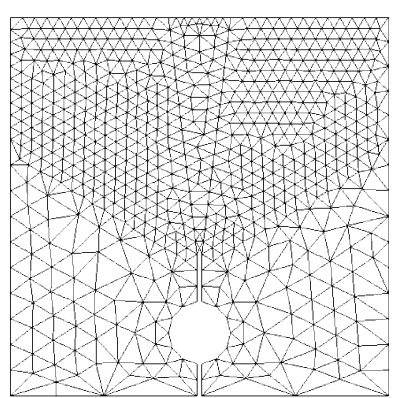

(c)

Finit element mesh: 1332 enhanced linear triangles 2048 nodes
Experimental (Kobayashi et al. 1985)

- Strong discontinuity approach

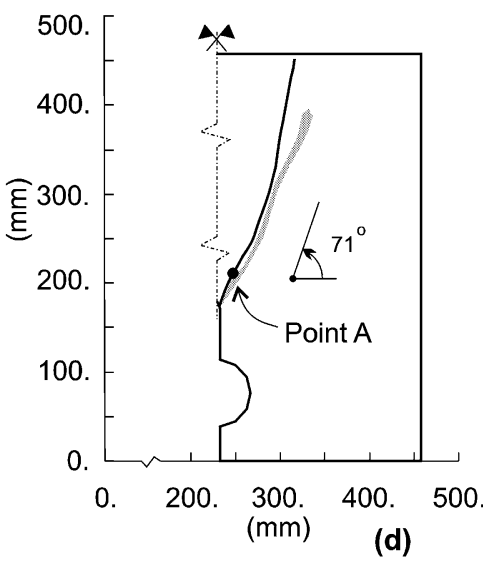

Fig. 8. Notched concrete specimen: (a)-(c) geometrical and loading data, (d) crack trajectories.
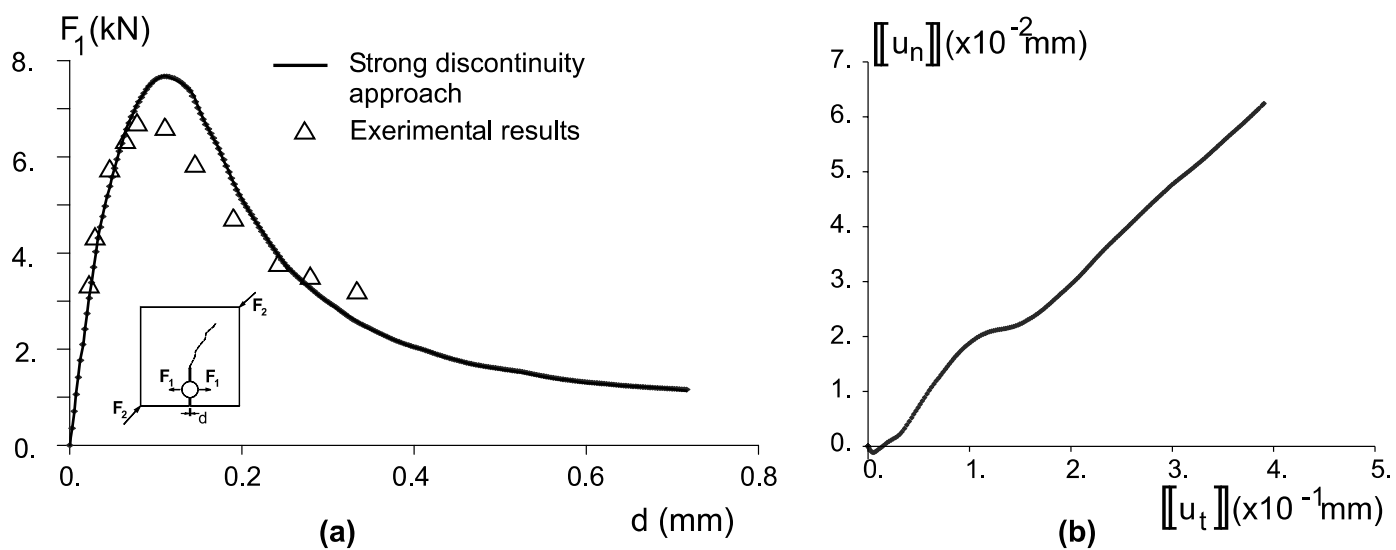

Fig. 9. Notched concrete specimen: (a) force $F_{1}$ vs. CMOD, (b) normal displacement jump, $\llbracket u \rrbracket_{n}$, vs. tangential displacement jump, $\llbracket u \rrbracket_{t}$, at point A.

develops from the notch tip with an inclination angle of $71^{\circ}$ with respect to the vertical (see Fig. 8(d)). For the numerical simulation the following material data have been adopted: $E=30.5 \mathrm{GPa}, v=0.2, G_{\mathrm{f}}=100$ $\mathrm{N} / \mathrm{m}$ and $\sigma_{u}=3 \mathrm{MPa}$. The width of the specimen is $t=50.8 \mathrm{~mm}$.

The finite element mesh used for the analysis is shown in Fig. 8(c). The obtained numerical results are compared with the experimental ones in terms of the crack mouth opening displacement (CMOD), $d$ in Fig. 9(a), and in terms of the crack path in Fig. 8(d). In Fig. 9(b) the normal $\left(\llbracket u \rrbracket_{n}\right)$ vs. the tangential $\left(\llbracket u \rrbracket_{t}\right)$ displacement jump at point A of Fig. 8(d) are plotted, stating clearly the mixed mode character $\left(\llbracket u \rrbracket_{n} \neq 0\right.$, $\llbracket u \rrbracket_{t} \neq 0$ ) of the resulting crack. 

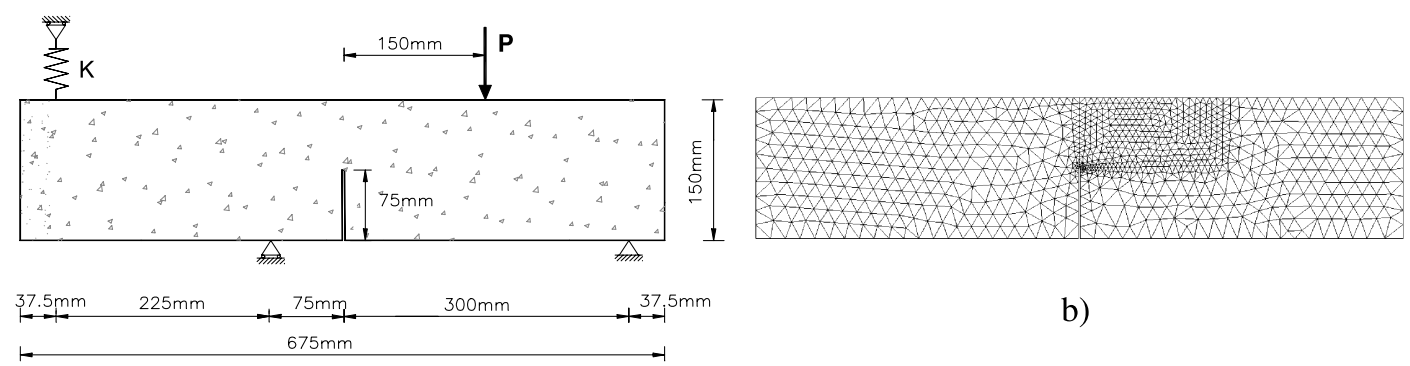

b)

a)

Fig. 10. Three and four points bending tests: $K=0$ for three points bending test and $K=\infty$ for four points bending test: (a) geometry; (b) finite element mesh.

\subsection{Three and four points bending tests}

The experimental results reported in Ref. [9], in which a novel testing procedure for mixed mode crack propagation on concrete under non-proportional loading was developed, were numerically reproduced using the SDA. Fig. 10(a) shows the geometry of two different test specimens, depending on a spring boundary condition. A spring stiffness value $(K)$ equal to zero, $K=0$, represents a three points bending test whereas $K=\infty$ represents a four points bending test. The thickness of the specimens is $t=50 \mathrm{~mm}$, and the corresponding finite element mesh (1600 enhanced linear triangle elements) is presented in Fig. 10(b). The considered material parameters are: $E=38 \mathrm{GPa}, v=0.18, \sigma_{u}=3.0 \mathrm{MPa}$ and $G_{\mathrm{f}}=69 \mathrm{~N} / \mathrm{m}$. These are again mixed mode fracture cases leading to numerical crack paths that match very well the experimental ones as it is shown in Fig. 11(a) (notice that different spring stiffness values $(K)$ cause different crack trajectories). An amplification of the deformed set of elements that capture the discontinuity near the notch is also shown in Fig. 11(b).

In Fig. 11(c)-(d) load $P$ vs. CMOD (crack mouth opening displacement) curves obtained in the numerical simulation are shown to fit very well into the experimental band of results. Finally, in Fig. 12(a) the tangential displacement jump vs. normal displacement jump curve shows the mixed mode character of the fracture.

In Fig. 12(b) the $\sigma_{x x}-\llbracket u \rrbracket_{n}$ (horizontal stress vs. normal jump) trajectories, at the tip of the notch, are plotted for a cyclic loading case in which the loading is reversed at a certain point $\mathrm{B}$, to be completely released at point $\mathrm{C}$, and then reversed again to load the specimen up to the complete failure. As it is shown in the figure, the numerically response, obtained using the continuum damage model of Section 3.2, exactly fulfills what is expected for the induced discrete damage model of Fig. $3 \mathrm{~b}$ in terms of the traction-jump parameters. The crack onsets at point A, when the peak stress is reached, then loading (with softening) takes places up to point $\mathrm{B}$ when the load is reversed. Beyond that point, a damaged-elastic unloading takes places up to point $\mathrm{C}$ where the crack is completely closed and the stress is released $\left(\llbracket u \rrbracket_{n}=0, \sigma_{x x}=0\right)$. The subsequent reloading produces, first, an elastic behaviour up to point $\mathrm{B}$ and, then, the rest of the loading trajectory B-D. Notice that discrete damage model was never introduced in the analysis but, as predicted by the theoretical analysis, it is effectively reproduced by the SDA.

\subsection{Gravity dam model}

A reduced model of a concrete gravity dam tested by Carpintieri et al. [5] was analyzed using the tools of the SDA. The notched upstream face of the model was loaded with a pressure equivalent to a water 


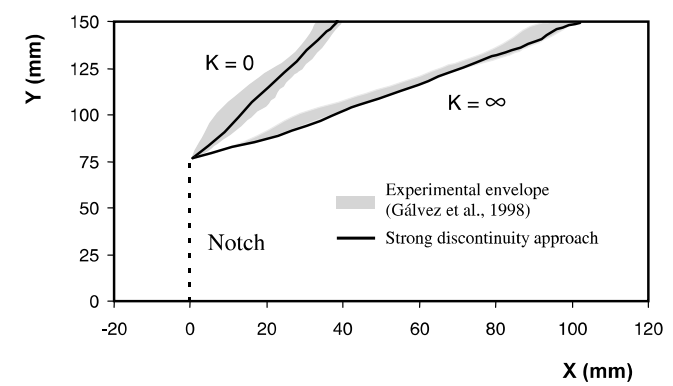

a)

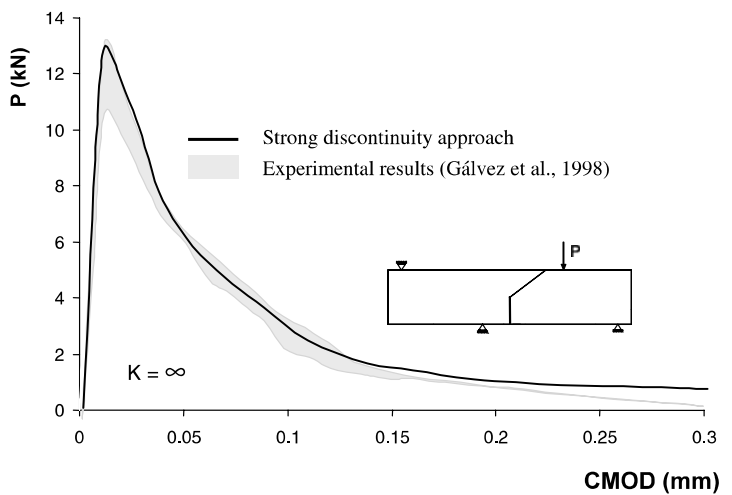

c)

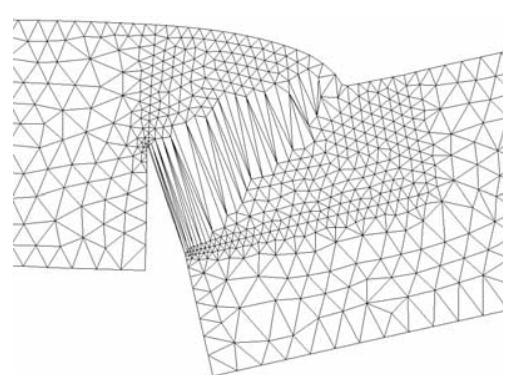

b)

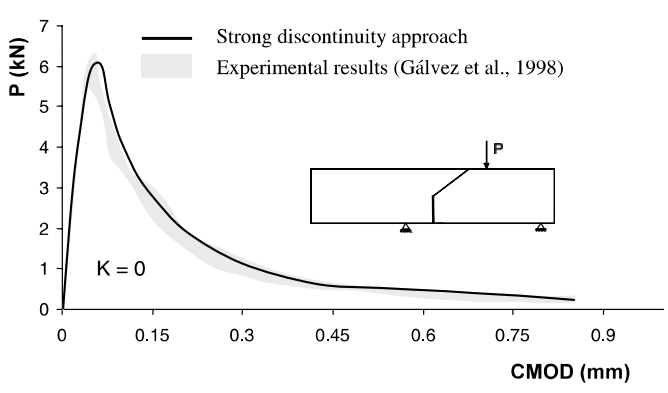

d)

Fig. 11. Three and four points bending tests: (a) experimental envelops and numerical predictions of crack trajectories, (b) amplification of the deformed mesh, (c,d) load P-CMOD responses.

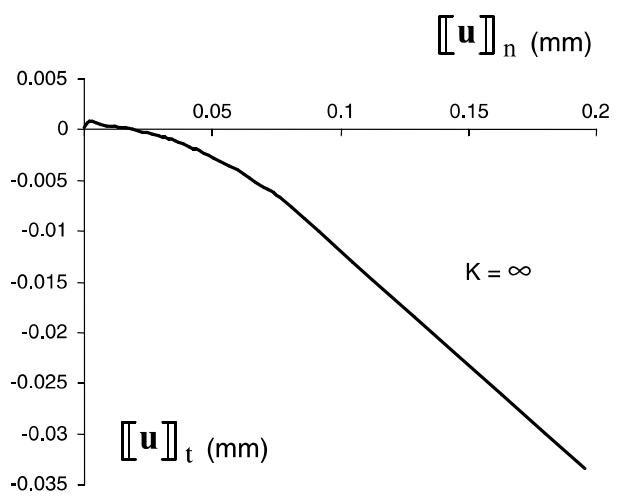

a)

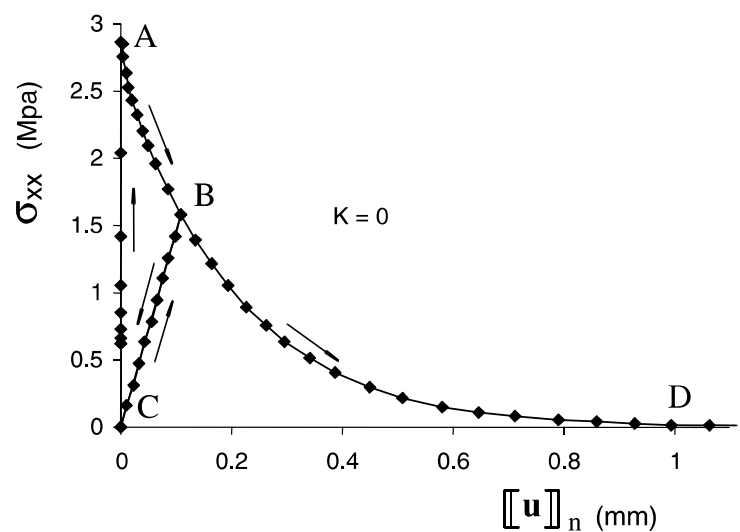

b)

Fig. 12. Three points bending test $(K=0)$ : (a) normal displacement jump $\llbracket u \rrbracket_{n}$ vs. tangential displacement jump $\llbracket u \rrbracket_{t}$, (b) tractiondisplacement jump trajectories at the tip of the notch. 


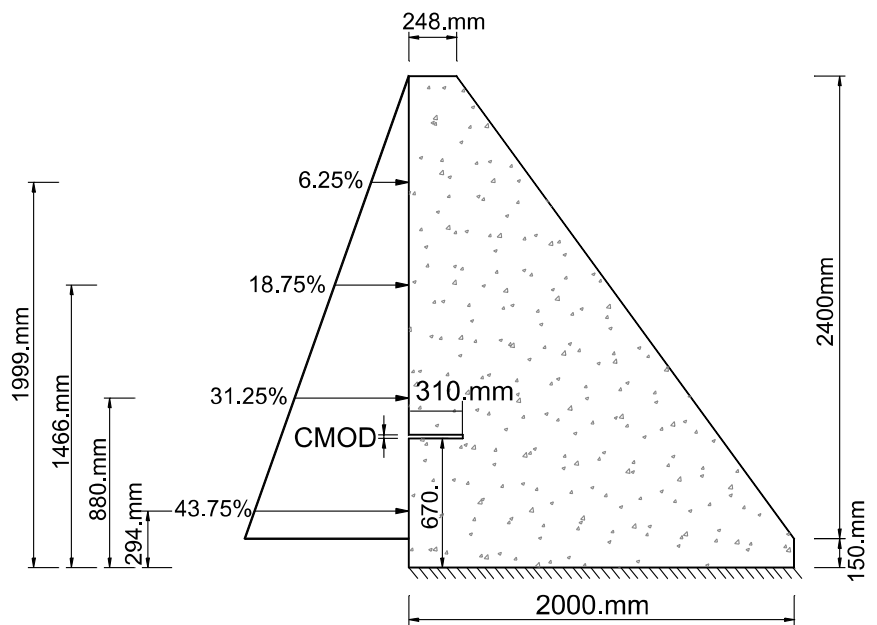

(a)

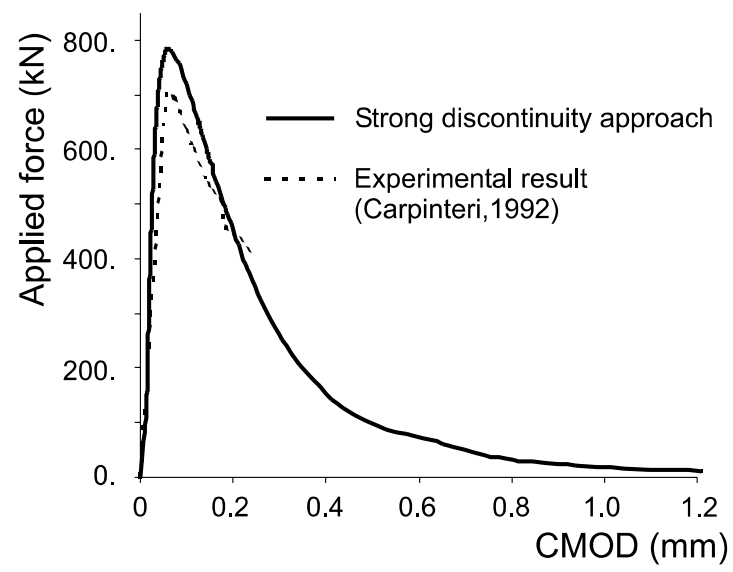

(c)

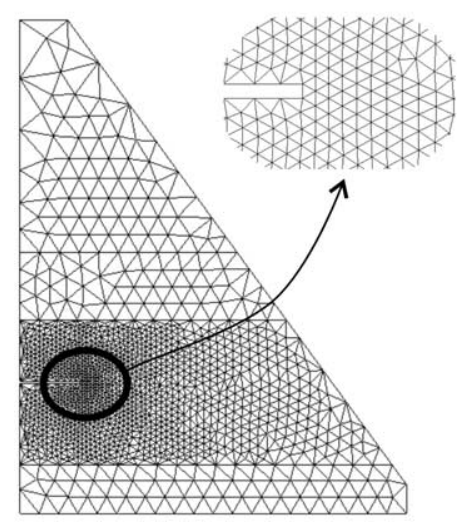

(b)

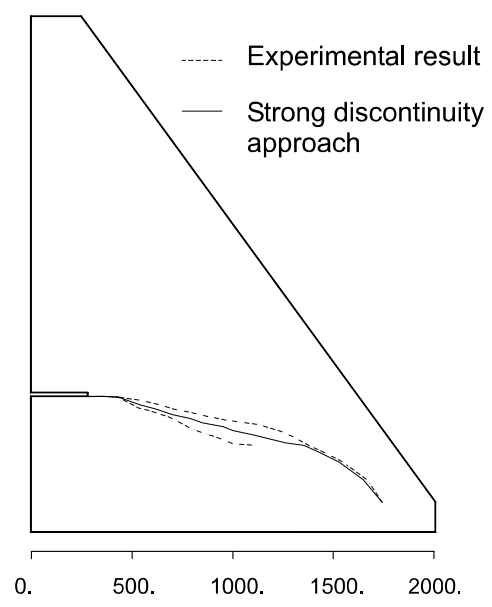

(d)

Fig. 13. Gravity dam model: (a) geometric definition, (b) finite element mesh, (c) total upstream face load vs. CMOD displacement, (d) crack trajectory.

column, as it is indicated in Fig. 13(a), in order to induce a curved crack that propagates from the tip of the notch towards the downstream face (see Fig. 13(d)). The numerical simulation is done on the basis of the SDA, with the continuum damage model of Section 3.2, and the finite element mesh of Fig. 13(b). The considered material parameters are: $E=35700 \mathrm{MPa}, v=0.18, \sigma_{u}=3.6 \mathrm{MPa}$ and $G_{\mathrm{f}}=184 \mathrm{~N} / \mathrm{m}$. Although the depth of the notch was not reported in the reference, for the numerical simulation it was taken $t=310$ $\mathrm{mm}$.

In Fig. 13(c) and (d) the numerical and experimental results, in terms of the resulting total up stream face load vs. CMOD curves (Fig. 13(c)) and the crack trajectories (Fig. 13(d)) are presented. In both cases, the numerical results provided by the strong discontinuity approach show good agreement with the experimental ones. Notice that the curved character of the crack trajectories is correctly captured by the SDA simulation. 

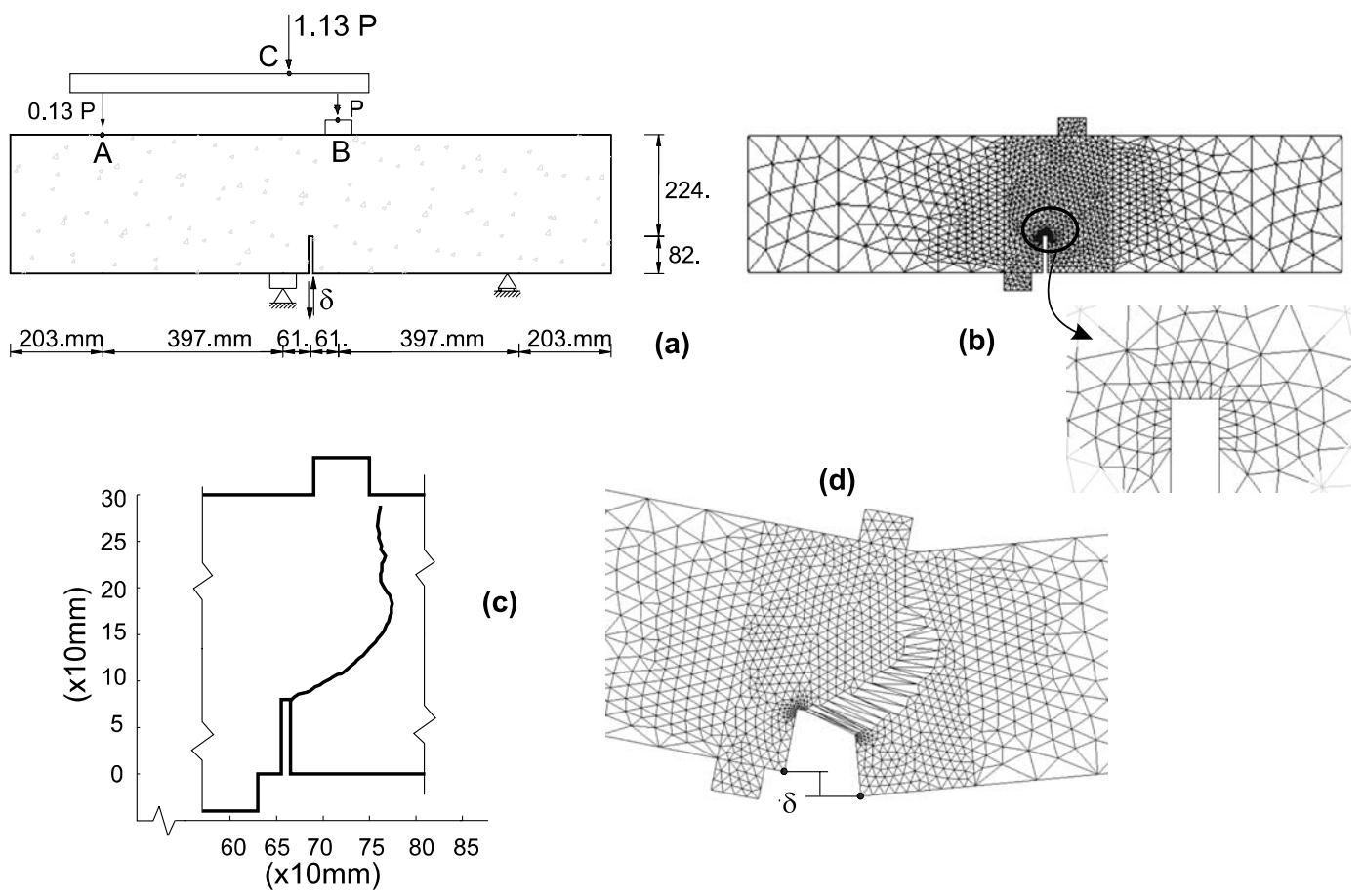

Fig. 14. Four points bending test: (a) geometrical properties, (b) finite element mesh, (c) crack trajectory (d) finite element localization pattern.

\subsection{Four points bending test}

We shall deal now with the classical four points bending tests, on a concrete beam, reported by Arrea and Ingraffea in 1982 [2]. The main difference with the ones considered in Section 4.2 is that in this case the external load is applied through a flexible steel beam which is responsible for a curved crack trajectory. The geometry of the problem is sketched in Fig. 14(a), where the thickness is $t=140 \mathrm{~mm}$, and the corresponding finite element mesh (1997 enhanced linear triangle elements) is presented in Fig. 14(b). The considered material parameters are $E=24800 \mathrm{MPa}, v=0.18, \sigma_{u}=2.8 \mathrm{MPa}$ and $G_{\mathrm{f}}=100 \mathrm{~N} / \mathrm{m}$. The SDA simulation results in a curved crack trajectory that agrees very well with the experimental one shown in Fig. 14(c). An amplification of the deformed set of elements that capture the discontinuity near the notch is also shown in Fig. 14(d).

In Fig. 15(a) the load, $P$ vs. the crack mouth sliding displacement (CMSD) ( $\delta$ in Fig. 14(a)) curve is shown to fit into the experimental band of results. Finally, in Fig. 15(b) the typical load-deflection, at point C of Fig. 14(a), curve exhibiting the classical snapback response, is also presented.

\section{Concluding remarks}

In the preceding sections it has been shown that displacement discontinuities (fractures, cracks etc.) can be modelled from the continuum mechanics side through the so called SDA. In essence, the approach owns a continuum character since, for analysis and modelling purposes, it keeps the continuum format for both 


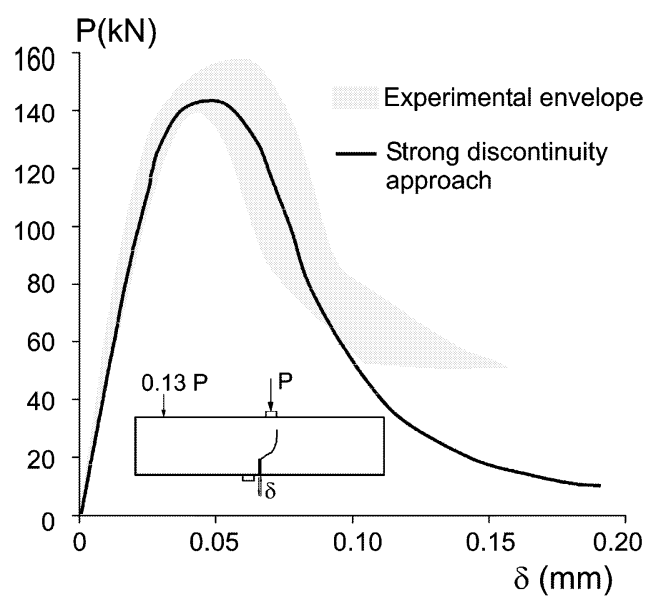

(a)

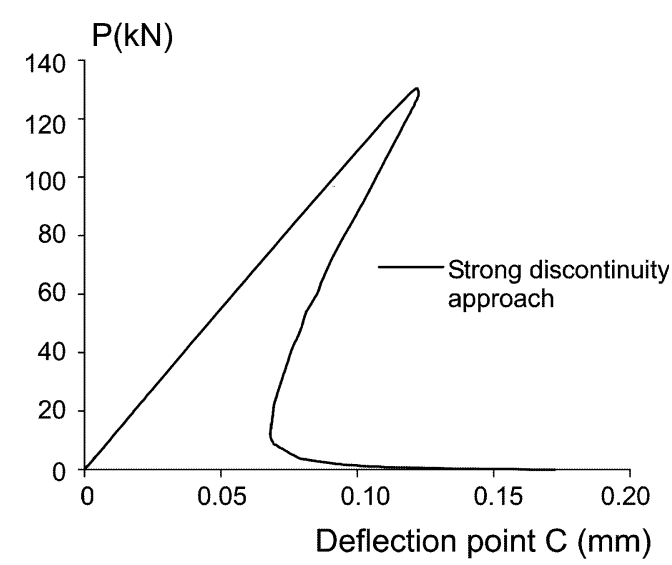

(b)

Fig. 15. Four points bending test: (a) load vs. CMSD curve and experimental band of results, (b) load-deflection curve.

the kinematics and the constitutive equation. However it has been proved that the introduction of two main ingredients i.e.

- the regularized discontinuous kinematics (and the associated weak and strong discontinuities),

- the regularization of the softening law,

induces, for every parent continuum constitutive model, a discrete counterpart that is implicitly fulfilled at the discontinuity interface and provides the tractions (continuous across the interface) in terms of the displacement jumps. The resulting discrete (traction-jump) constitutive model is equipped with ingredients such as free energy description, internal variables evolution and loading-unloading conditions, all of them determined in terms of the appropriated projections of the parent continuum constitutive model onto the discontinuity interface. Also it inherits the family properties (damage, elasto-plastic character) of the parent model. Besides, some fracture-mechanics-type material properties, as the fracture energy, can be straightforwardly recovered from the material properties of the continuum model like the peak stress and the softening parameter.

Therefore, the strong discontinuity approach provides the intended link with the non-linear decohesive fracture mechanics, where the aforementioned discrete constitutive equations were first developed, and the non-linear continuum mechanics. However, it has to be stressed that for modelling purposes the analysis is formally held in a continuum environment this providing the advantageous possibility to keep the whole analysis and simulation (both the continuous and discontinuous regimes) in the continuum format.

Although for the simple 1D cases there is no restriction for the induction of strong discontinuities by discontinuous bifurcation of the stress-strain field at the peak stress, for the general 2D-3D case the panorama is slightly different. In fact, it is found that some strong discontinuity conditions on the stress field have to be fulfilled at the strong discontinuity regime and, therefore, they preclude the strong discontinuity to originate directly from a discontinuous bifurcation. This drawback can be overcome by considering the weak discontinuity kinematics and the associated weak discontinuity regime, in between the bifurcation and the strong discontinuity regime, as an intermediate stage characterized by the bandwidth evolution from a bifurcation value to zero. Therefore, from the mathematical point of view, the goal of the weak discontinuity regime is to induce the strong discontinuity conditions. This can be translated to the 
physical viewpoint by considering the weak discontinuity as a fracture process regime, which is devoted to the induction of the actual fracture, and the zone where it takes places as the fracture process zone, another typical ingredient of the non-linear fracture mechanics [4] recovered by the strong discontinuity approach that closes the link.

Regarding the fracture range, there is no conceptual restriction for the SDA to tackle both brittle or ductile cases. Although, for the sake of simplicity, this point has not been considered here, modelling of ductile fracture can be done by resorting to a longer prebifurcation non-linear behaviour which, in turn, can require the continuum model to be equipped with large-strain kinematics. This has already been done in recent works $[1,14,23]$.

Although distinction on fracture modes (mode I, mode II or mixed mode) is not a priori done in the SDA, not any continuum constitutive model is appropriate to model any fracture process. For instance, it can be shown that a J2 (von Mises) continuum plasticity model in plain-strain is only compatible with tangential displacement jumps (mode II case) [19]. However, the same model in plain-stress cases sets no restriction on the type of displacement jump. Therefore, an appropriate choice of the continuum constitutive model family is also necessary in the SDA to succeed in modelling the fracture behaviour of the material.

\section{Acknowledgements}

The second author acknowledges the Spanish Ministry of Education and Culture through the S.E.U.I (Secretaría de Estado de Universidades e Investigación) for their economical support to this research. Also the fourth author acknowledges CAPES (Brazil) for its financial support.

\section{References}

[1] Armero F, Garikipati K. An analysis of strong discontinuities in multiplicative finite strain plasticity and their relation with the numerical simulation of strain localization in solids. Int J Sol Struct 1996;33(20-22):2863-85.

[2] Arrea M, Ingraffea AR. Mixed-mode crack propagation in mortar and concrete. Technical report 81-13, Department of Structural Engineering, Cornell University, New York, 1982.

[3] Bazant ZP. Crack band theory for fracture of concrete. Materiaux et Constructions 1983;93:155-77.

[4] Bazant ZP, Planas J. Fracture and size effect in concrete and other quasibrittle materials. Boca Raton: CRC Press; 1998.

[5] Carpintieri A, Valente SV, Ferrara G, Imperato L. Experimental and numerical fracture modelling of a gravity dam. In: Bazant ZP, editor. Fracture mechanics of concrete structures. 1992. p. 351-60.

[6] de Borst R, Sluys LJ, Muhlhaus HB, Pamin J. Fundamental issues in finite element analyses of localization of deformation. Engng Computat 1993;10:99-121.

[7] Dvorkin EN, Cuitino AM, Gioia G. Finite elements with displacement embedded localization lines insensitive to mesh size and distortions. Int J Numer Meth Engng 1990;30:541-64.

[8] Faria R, Oliver J, Cervera M. A strain-based plastic viscous-damage model for massive concrete structures. Int J Sol Struct 1998;(14):1533-58.

[9] Galvez JC, Elices M, Guinea GV, Planas J. Mixed mode fracture of concrete under proportional and nonproportional loading. Intl J Fract 1998;94:267-84.

[10] Hillerborg A. Numerical methods to simulate softening and fracture of concrete. In: Sih GC, Di Tomaso A, editors. Fracture mechanics of concrete: structural application and numerical calculation. 1985. p. 141-70.

[11] Jirasek M. Finite elements with embedded cracks. Internal Report - Ecole Polytechnique Federale de Lausanne, LSC Internal Report 98/01, April 1998.

[12] Kobayashi AS, Hawkins MN, Barker DB, Liaw BM. Fracture process zone of concrete. In: Shah SP, editor. Application of fracture mechanics to cementitious composites, Dordrecht: Marinus Nujhoff Publications; 1985. p. 25-50.

[13] Larsson R, Runesson K, Sture S. Embedded localization band in undrained soil based on regularized strong discontinuity theory and finite element analysis. Int J Sol Struct 1996;33(20-22):3081-101. 
[14] Larsson R, Steinman P, Runesson K. Finite element embedded localization band for finite strain plasticity based on a regularized strong discontinuity. Mech Cohesive Frictional Mater 1998;4:171-94.

[15] Needleman A. Material rate dependence and mesh sensitivity in localization problems. Comp Meth Appl Mech Engng 1988;67:69-85.

[16] Oliver J. A consistent characteristic length for smeared cracking models. Int J Num Meth Engng 1989;28:461-74.

[17] Oliver J. Modeling strong discontinuities in solid mechanics via strain softening constitutive equations. Part I: fundamentals. Int J Num Meth Engng 1996;39(21):3575-600.

[18] Oliver J. Modeling strong discontinuities in solid mechanics via strain softening constitutive equations. Part 2: numerical simulation. Int J Num Meth Engng 1996;39(21):3601-23.

[19] Oliver J. On the discrete constitutive models induced by strong discontinuity kinematics and continuum constitutive equations. Int J Sol Struct 2000;37:7207-29.

[20] Oliver J, Cervera M, Manzoli O. On the use of J2 plasticity models for the simulation of 2D strong discontinuities in solids. In: Owen DRJ, Onate E, Hinton E, editors. Proceedings of the International Conference on Computational Plasticity, Barcelona, Spain, 1997. p. 38-55.

[21] Oliver J, Cervera M, Manzoli O. On the use of strain-softening models for the simulation of strong discontinuities in solids. In: de Borst R, van der Giessen E, editors. Material instabilities in solids. Wiley: New York; 1998. p. 107-123 [chapter 8].

[22] Oliver J, Cervera M, Manzoli O. Strong discontinuities and continuum plasticity models: the strong discontinuity approach. Int J Plast 1999;15(3):319-51.

[23] Oliver J, Pulido MDG. On the use of strain-softening damage constitutive equations to model cracking of concrete. In: de Borst R, Bicanic N, Mang HA, Meschke G, editors. Proceedings EURO-C 1998, Computational modeling of concrete structures. Balkema, 1998. p. 363-72.

[24] Oliver J, Simo J. Modelling strong discontinuities by means of strain softening constitutive equations. In: Mang H, et al., editors, Proceedings EURO-C 1994, Computer modeling of concrete structures. Pineridge Press, Swansea, 1994. p. 363-72.

[25] Planas J, Elices M, Guinea GV. Cohesive cracks versus nonlocal models: closing the gap. Int J Fract 1993;63:173-87.

[26] Regueiro RA, Borja RI. A finite element model of localized deformation in frictional materials taking a strong discontinuity approach. Finite Element Anal Design 1999;33:283-315.

[27] Rots JG. Computational Modeling of Concrete Fractures, Delft University of Technology, 1988. PhD thesis.

[28] Runesson K, Ottosen NS, Peric D. Discontinuous bifurcations of elastic-plastic solutions at plane stress and plane strain. Int J Plast 1991;7:99-121.

[29] Simo J, Oliver J. A new approach to the analysis and simulation of strong discontinuities. In: Bazant ZP, et al., editor, Fracture and damage in quasi-brittle structures, 1994. p. 25-39.

[30] Simo J, Oliver J, Armero F. An analysis of strong discontinuities induced by strain-softening in rate-independent inelastic solids. Computat Mech 1993;12:277-96.

[31] Steinmann P, Willam K. Finite element analysis of elastoplastic discontinuities. J Engng Mech 1994;120:2428-42.

[32] Wells GN, Sluys LJ. Application of embedded discontinuities for softening solids. Engng Fract Mech 2000;65:263-81.

[33] Wells GN, Sluys LJ. Application of continuum laws in discontinuity analysis based on a regularized displacement discontinuity. In: ECCM'99, European Conference on Computational Mechanics, August 31-September 3, 1999.

[34] Willam K, Sobh N. Bifurcation analysis of tangential material operators. In: Pande GN, Middleton J, editors. Transient dynamic analysis and constitutive laws for engineering materials, vol. 2, Martinus: Nijhoff Publishers; 1987. p. C4/1-13. 